\title{
Spines, Heegaard Splittings and the Reidemeister-Turaev Torsion
}

\author{
Yuya KODA
}

Keio University

(Communicated by Y. Maeda)

\begin{abstract}
In this article, we introduce a formula for the Reidemeister-Turaev torsion $\tau^{\varphi}\left(M,[\mathcal{V}], o_{M}\right)$ of an arbitrary closed 3-manifold $M$ equipped with a $\operatorname{Spin}^{c}$ structure [V $]$. As a CW-structure of $M$ needed in the process of the computation, we adopt the one induced from a Heegaard splitting which is compatible, via the concept of flow-spine, with a given $\operatorname{Spin}^{c}$ structure.
\end{abstract}

\section{Introduction}

Reidemeister torsion is a very important invariant of smooth 3-manifolds which has useful applications in knot theory, quantum field theory, dynamical systems and so on. This invariant $\tau^{\varphi}(M)$ of a manifold $M$ is defined up to multiplication by $\pm \varphi\left(\pi_{1}(M)\right)$, where $\varphi$ is a representation of the integral group ring $\mathbf{Z}\left[\pi_{1}(M)\right]$ to a field $F$. To remove this ambiguity, Turaev introduced in [14] an idea of Euler structure which is represented as a homology class of non-singular vector fields on a given manifold and equivalent to Spinc structure in the 3-dimensional case. Turaev's refined Reidemeister torsion $\tau^{\varphi}\left(M,[\mathcal{V}], \mathfrak{o}_{M}\right)$, which we call the Reidemeister-Turaev torsion, is interpreted as an invariant of 3-manifolds equipped with $\operatorname{Spin}^{c}$ structures. This invariant has been recognized to have strong connections with many developments in 3-dimensional geometry and topology: Seiberg-Witten invariant (cf. $[8,15])$, Heegaard Floer homology ( $c f$. [10,11]) etc.

The only well-known method for calculating the Reidemeister-Turaev torsion is given by using the gluing formula along boundary tori (eg. formulae for Dehn surgeries and graph manifolds, (cf. $[9,17,18])$. In this paper we give a way to compute this invariant, which is compatible with Heegaard splittings.

To study 3-manifolds with $\operatorname{Spin}^{c}$ structures, we review a branched surface $P$ called a flow-spine $(c f .[1,5])$, which is embedded in a 3-manifold $M$ naturally carrying a vector field $\mathcal{V}$ on $M$ and whose complement $M \backslash P$ is an open 3-ball $B$. By cutting the 3-manifold $M$ along the flow-spine $P$ we get a presentation of $M$ as a ball $B$ with an equivalence relation $\sim$ on the boudary 2-sphere $\partial B=S^{2}$ satisfying $M=B / \sim$ and $P=\partial B / \sim$, which we call a $D S$-diagram (or a spinal presentation) (cf. for example $[1,3,4,5,12])$. Then we introduce a

Received February 27, 2006; revised August 23, 2006 
method for calculating the Reidemeister-Turaev torsion using the cellular decomposition derived from a Heegaard splitting, here to have this Heegaard splitting coherent to a given $\operatorname{Spin}^{c}$ structure $[\mathcal{V}]$, we construct it from a flow-spine corresponding to the non singular vector field $\mathcal{V}$. As a consequence, we get an explicit formula of the Reidemeister-Turaev torsion which covers all 3-manifolds equipped with $\operatorname{Spin}^{c}$ structures, see Theorem 7.8. In the final part of this paper, we give some examples of computations. We explain how to use this invariant and give also an example of computation of the Seiberg-Witten invariant. Our argument in this paper will freely traverse the viewpoints of both the smooth and the PL categories.

\section{Review on Reidemeister torsion}

1.1. The Reidemeister torsions of manifolds. Let $F$ be a field and let $E$ be an $n$ dimensional vector space over $F$. For two ordered bases $r=\left(r_{1}, \ldots, r_{n}\right)$ and $s=\left(s_{1}, \ldots, s_{n}\right)$ of $E$, we write $[r / s]=\operatorname{det}\left(a_{i j}\right) \in F^{\times}$, where $r_{i}=\sum_{j=1}^{n} a_{i j} s_{j}$. The bases $r$ and $s$ are said to be equivalent if $[\mathrm{r} / \mathrm{s}]=1$.

Let $C=\left(0 \stackrel{\partial_{m}}{\longrightarrow} C_{m} \stackrel{\partial_{m-1}}{\longrightarrow} C_{m-1} \rightarrow \cdots \rightarrow C_{1} \stackrel{\partial_{0}}{\rightarrow} C_{0} \stackrel{\partial_{-1}}{\longrightarrow} 0\right)$ be a finite dimensional chain complex over $F$. For each $0 \leq i \leq m$, set $B_{i}=\operatorname{Im} \partial_{i}, Z_{i}=\operatorname{Ker} \partial_{i-1}$ and $H_{i}=Z_{i} / B_{i}$. The chain complex is said to be acyclic if $H_{i}=0$ for all $i$. Suppose that $C$ is acyclic and $C_{i}$ is endowed with a distinguished basis $c_{i}$ for each $i$. Choose an ordered set of vectors $b_{i}$ in $C_{i}$ for each $i$ such that $\partial_{i-1}\left(b_{i}\right)$ forms a basis of $B_{i-1}$. By the above construction, $\partial_{i}\left(b_{i+1}\right)$ and $b_{i}$ are combined to be a new basis $\partial_{i}\left(b_{i+1}\right) b_{i}$ of $C_{i}$. With this notation, the torsion of $C$ is defined by

$$
\tau(C)=\prod_{i=0}^{m}\left[\partial_{i}\left(b_{i+1}\right) b_{i} / c_{i}\right]^{(-1)^{i+1}} \in F^{\times} .
$$

Let $M$ be a compact connected orientable smooth manifold of an arbitrary dimension. Let $X$ be a CW-decomposition of $M, p: \hat{X} \rightarrow X$ be its maximal abelian cover and $F$ be a field. We can equip $\hat{X}$ with the $\mathrm{CW}$-structure naturally induced by that of $X$, and then we regard $C_{*}(\hat{X})$ as a left $\mathbf{Z}\left[H_{1}(X)\right]$-module via the monodromy. Let $\left\{e_{i}^{k}\right\}$ be the set of all oriented $k$-cells in $X$. A family of cells of $\hat{X}$ is said to be fundamental if over each cell of $X$ exactly one cell of this family lies. When we choose a fundamental family $\left\{\hat{e}_{i}^{k}\right\}$ of cells of $\hat{X}$ and orient and order these cells in arbitrary way, it becomes a free $\mathbf{Z}\left[H_{1}(X)\right]$-basis of $C_{k}(\hat{X})$. (i.e. $\left.C_{k}(\hat{X})=\bigoplus_{i} \mathbf{Z}\left[H_{1}(X)\right] \hat{e}_{i}^{k}\right)$. In this way, we can regard $C_{*}(\hat{X})$ as a chain complex with basis.

Let $\varphi: \mathbf{Z}\left[H_{1}(X)\right] \rightarrow F$ be a ring homomorphism. If the based chain complex $C_{*}^{\varphi}(X)=$ $F \otimes_{\varphi} C_{*}(\hat{X})$ over $F$ is acyclic, the Reidemeister torsion of $X$ is

$$
\tau^{\varphi}(M)=\tau\left(C_{*}^{\varphi}(X)\right) \in F^{\times} / \pm \varphi\left(H_{1}(M)\right) .
$$

Otherwise, set $\tau^{\varphi}(M)=0 \in F$. 
1.2. Turaev's refinement of the torsions with $\operatorname{Spin}^{c}$ structures. Let $X$ be a finite connected CW-complex. An Euler chain is a one dimensional singular chain $\theta$ in $X$ with $\partial \theta=\sum_{a}(-1)^{\operatorname{dim} a} \sigma_{a}$, where $a$ runs over all cells of $X$ and $\sigma_{a}$ is the barycenter of $a$. Two Euler chains $\theta_{1}$ and $\theta_{2}$ are said to be homologous if the chain $\theta_{1}-\theta_{2}$ is a boundary. A combinatorial Spin ${ }^{c}$ structure is a homology class of Euler chains. We denote by $\operatorname{Eul}(X)$ the set of combinatorial $\operatorname{Spin}^{c}$ structures. Remark that $\chi(X)=0$ implies that $\operatorname{Eul}(X)$ is not void. Note that $H_{1}(X)$ acts freely and transitively on $\operatorname{Eul}(X)$ in the natural way, see [14].

An important example of Euler chain is a spider, which consists of a base point $\sigma$ and (oriented) paths from $\sigma$ to $\sigma_{a}$ for each even dimensional cell $a$ and paths from $\sigma_{a}$ to $\sigma$ for each odd dimensional cell $a$. When we fix a lift $\hat{\sigma}$ of $\sigma$ to $\hat{X}$, we can naturally define the fundamental family of cells for each combinatorial $\operatorname{Spin}^{c}$ structure using its spider. Conversely, any combinatorial $\operatorname{Spin}^{c}$ structure on $X$ arises from a fundamental family of cells in $\hat{X}$.

Each cellular subdivision $X^{\prime}$ of $X$ defines a canonical $H_{1}(X)$-equivariant bijection $\Psi_{X, X^{\prime}}: \operatorname{Eul}(X) \rightarrow \operatorname{Eul}\left(X^{\prime}\right)$. We can describe this bijection as follows: for a fundamental family $S$ of cells in $\hat{X}$, the cells of $\hat{X}^{\prime}$ lying in $\cup_{a \in S} a$ form a fundamental family $S^{\prime}$, then the element of $\operatorname{Eul}(X)$ arising from $S$ is mapped to the element of $\operatorname{Eul}\left(X^{\prime}\right)$ arising from $S^{\prime}$. This observation admits us to define the combinatorial $\operatorname{Spin}^{c}$ structures on a 3-manifold $M$ by taking arbitrary cellular decomposition $X$ of $M$.

Turaev's refinement of the Reidemeister torsion is based on the two notions, combinatorial Spin ${ }^{c}$ structure and homology orientation (this is an orientation of the real vector space $\left.H_{*}(M ; \mathbf{R})\right)$. A combinatorial $\operatorname{Spin}^{c}$ structure distinguishes an equivalent class of the bases of the twisted chain complex up to order as we explained above, and a homology orientation gives a way to account for the sign indeterminacy inherent in ignoring order above, see [18]. Let $M$ be a compact manifold with CW-structure $X$. Let $F$ be a field and $\varphi: \mathbf{Z}\left[H_{1}(M)\right] \rightarrow F$ be a ring homomorphism. For a combinatorial $\operatorname{Spin}^{c}$ structure $\theta \in \operatorname{Eul}(X)$ and a homology orientation $\mathfrak{o}$, the Reidemeister-Turaev torsion $\tau^{\varphi}(M, \theta, \mathfrak{o}) \in F$ is defined to be a torsion of the twisted chain complex with an ordered basis corresponding to $\theta$ and $\mathfrak{o}$.

Let $M$ be a closed smooth 3-manifold. Two non-singular vector fields $\mathcal{V}_{1}$ and $\mathcal{V}_{2}$ on $M$ are said to be homologous if there exists a closed 3-ball $B \subset M$ such that the restrictions of $\mathcal{V}_{1}$ and $\mathcal{V}_{2}$ to $M \backslash \operatorname{Int}(B)$ are homotopic as non-singular vector fields. A smooth Spinc structure is a homology class of non-singular vector fields. We denote by $\operatorname{Vect}(M)$ the set of smooth $\operatorname{Spin}^{c}$ structure on $M$. The action of $H_{1}(M)$ to $\operatorname{Vect}(M)$ is defined through Reeb surgery, see $[18,19]$ for details.

THEOREM 1.1 (Turaev [14])). There is a canonical $H_{1}(M)$-equivariant bijection

$$
\Phi: \operatorname{Eul}(M) \rightarrow \operatorname{Vect}(M)
$$

Let $M$ be a closed oriented smooth 3-manifold with a CW-decomposition $X$. Recall that the intersection pairing $H_{i}(M ; \mathbf{R}) \times H_{3-i}(M ; \mathbf{R}) \rightarrow \mathbf{R}$ defined by the orientation of $M$ induces the canonical homology orientation $\mathfrak{o}_{M}$. Let $F$ be a field and $\varphi: \mathbf{Z}\left[H_{1}(M)\right] \rightarrow F$ 
be a ring homomorphism. For each smooth $\operatorname{Spin}^{c}$ structure $[\mathcal{V}] \in \operatorname{Vect}(M)$, the ReidemeisterTuraev torsion of the triple $\left(M,[\mathcal{V}], \mathfrak{o}_{M}\right)$ is defined by

$$
\tau^{\varphi}\left(M,[\mathcal{V}], \mathfrak{o}_{M}\right)=\tau^{\varphi}\left(M, \Phi^{-1}([\mathcal{V}]), \mathfrak{o}_{M}\right) \in F .
$$

In [2], Benedetti and Petronio described the inverse map $\Phi^{-1}$ using the notion of branched standard spines.

\section{Flow-spines and DS-diagrams with E-cycle}

2.1. The definition of DS-diagrams with E-cycle. In this section, we recall the notion of fake surfaces, flow-spines, DS-diagrams with E-cycle and blocks introduced in [4, 5, $6,1,3]$. See these papers for the precise definitions.

Let $M$ be a closed orientable 3-manifold. A singular surface $P$ in $M$ is a fake surface if each point of it has a neighborhood belonging to one of the three types shown in Figure 1.

By triangulation we mean a cellular decomposition of a manifold which consists of tetrahedra, and allows self-adjacencies and multiple adjacencies of tetrahedra.

A fake surface $P \subset M$ is called a simple spine if $M \backslash P$ is homeomorphic to an open 3ball. A spine $P$ is naturally stratified as $V(P) \subset S(P) \subset P$, where $V(P)$ is a set of vertices and $S(P)$ is the singular set. A branched spine $P$ is said to be standard if this stratification induces a $\mathrm{CW}$-decomposition of $P$. Remark that the dual decomposition of this cellular decomposition is a one-vertex triangulation of $M$.

We call a triple $\Delta=(G, \phi, P)$ a $D S$-diagram if it satisfies the following: (i) $G$ is a 3-regular graph (i.e. a graph, every vertex of which has degree 3 ) on a boundary of a closed 3-ball $B$; (ii) $P$ is a closed simple spine; (iii) $\phi: S^{2}=\partial B \rightarrow P$ is a local homeomorphism; and (iv) $\# \phi^{-1}(\phi(x))=2\left(x \in S^{2} \backslash G\right), \# \phi^{-1}(\phi(x))=3(x \in G \backslash V(G))$ and $\# \phi^{-1}(\phi(x))=$ $4(x \in V(G))$.

Given a DS-diagram $\Delta$, the quotient space $M(\Delta)=B / \phi$ becomes a closed (possibly non-orientable) 3-manifold. We call this manifold the realized manifold for $\Delta$, and conversely, $\Delta$ a DS-diagram of the 3-manifold $M(\Delta)$.

Let $\Delta=(G, \phi, P)$ be a DS-diagram. A cycle $e$ of $G$ is called an $E$-cycle if it satisfies the following conditions: (i) when we split the sphere as $S^{2} \backslash e=\Sigma_{1} \cup \Sigma_{2}$ (two open disks), it satisfies that $\#\left(e \cap \phi^{-1}(x)\right)=\#\left(\Sigma_{1} \cap \phi^{-1}(x)\right)=\#\left(\Sigma_{2} \cap \phi^{-1}(x)\right)=1$ for each point $x$ on

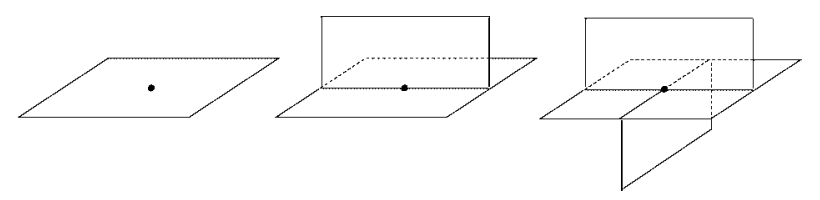

(i)

(ii)

(iii)

FIGURE 1. Neighborhoods of points in a fake surface. 
$S(P) \backslash V(P)$; (ii) \#( $\left.\Sigma_{1} \cap \phi^{-1}(x)\right)=\#\left(\Sigma_{2} \cap \phi^{-1}(x)\right)=1$ for each point $x$ on $P \backslash S(P)$; and

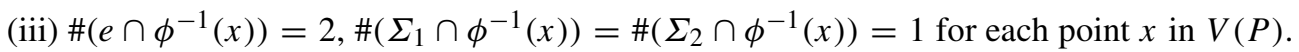
We denote by $\Delta(G, \phi, P ; e)$ a DS-diagram with E-cycle $e$. In this paper, every DS-diagram has an E-cycle.

Let $\Delta(G, \phi, P ; e)$ be a DS-diagram with E-cycle. We may assume that $B^{3}=\{(x, y, z) \in$ $\left.\mathbf{R}^{3} \mid x^{2}+y^{2}+z^{2} \leq 1\right\}, G \subset S^{2}=\partial B^{3}, e=S^{2} \cap\{z=0\}$. The sphere $S^{2}$ is divided by the E-cycle $e$ into two open disks $\Sigma_{+}=S^{2} \cap\{z>0\}, \Sigma_{-}=S^{2} \cap\{z<0\}$. The 3-ball $B^{3}$ is always assumed to be given the usual orientation so that it is compatible with the orientation of $S^{2}=\partial B^{3}$, on which an orientation was already fixed by the DS-diagram. We also assume that the orientation on the E-cycle $e$ is compatible with one on $S^{2}$ restricted to $\Sigma_{+}$. Then we can regard $M(\Delta)$ as an oriented 3-manifold.

Let $\mathcal{V}_{1}, \mathcal{V}_{2}$ be non-singular (i.e. nowhere vanishing) vector fields on a closed 3-manifold $M$. We denote $\mathcal{V}_{1} \simeq \mathcal{V}_{2}$ if $\mathcal{V}_{1}$ and $\mathcal{V}_{2}$ are homotopic in the class of non-singular vector fields on $M$. Set $\langle\mathcal{V}\rangle=\left\{\mathcal{V}_{1} \mid \mathcal{V}_{1}\right.$ is a non-singular vector field on $M$ with $\left.\mathcal{V} \simeq \mathcal{V}_{1}\right\}$.

DEFINITION 2.1. A vector field on $M(\Delta)$ belonging to the homotopy class $\langle\mathcal{V}(\Delta)\rangle=$ $\langle(-\partial / \partial z) / \phi\rangle$ is called an accompanying vector field of $\Delta$.

Since we regard a DS-diagram $\Delta$ as a topological object, the vector field $\mathcal{V}(\Delta)$ is not well-defined but its homotopy class $\langle\mathcal{V}(\Delta)\rangle$ is well-defined, hence the smooth $\operatorname{Spin}^{c}$ structure $[\mathcal{V}(\Delta)]$ is also well-defined.

EXAMPLE 2.2. A DS-diagram with E-cycle of Lens space $L(3,1)$ and its accompanying vector field are drawn in the left-hand side of Figure 2. We usually draw this diagram as in the right one, that is, the graph on the upper-half part of the sphere $\partial B^{3}$. It is shown in [5] that we can reconstruct the whole diagram when we are given this diagram.

The above construction of the pair $(M(\Delta),\langle\mathcal{V}(\Delta)\rangle)$ has a universality due to Theorem 2.3. In fact, this type of DS-diagrams is obtained by cutting the 3-manifold along a flow-spines $P$ defined in these papers of Ishii, see also [1].
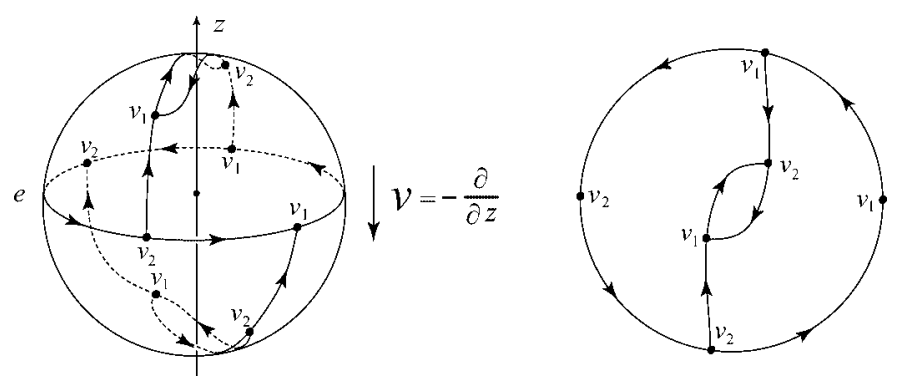

FIGURE 2. A DS-diagram with E-cycle of $L(3,1)$. 

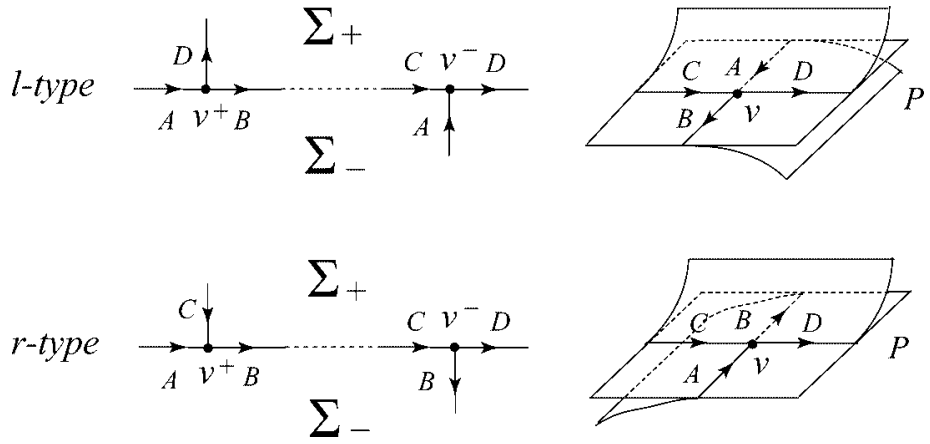

FIGURE 3. Two types of vertex $v$ and the corresponding branching structures of $P$.

THEOREM 2.3 (Ishii [5]). Let $M$ be a closed oriented 3-manifold and $\mathcal{V}$ be a nonsingular vector field on $M$. Then there exists such a DS-diagram $\Delta$ that admits a diffeomorphism $f: M(\Delta) \rightarrow M$ with $\langle\mathcal{V}\rangle=\left\langle f_{*}(\mathcal{V}(\Delta))\right\rangle$, where $f_{*}$ is the push-out induced from $f$.

For each vertex $v$ of the spine $P \subset M(\Delta)$, there are exactly two vertices $v^{+}$and $v^{-}$ on the E-cycle $e$ of the graph $G$ such that $\phi\left(v^{+}\right)=\phi\left(v^{-}\right)=v$. These two vertices are characterized by the condition whether the third edge, which connects to the vertex and is not on $e$, is on $\Sigma_{+}$or on $\Sigma_{-}$.

Each vertex $v \in V(P)$ is classified into the two types $l$ and $r$ shown in Figure 3. A code is a map $\gamma_{P}: V(P) \rightarrow\{l, r\}$ such that $\gamma_{P}(v)=l$ (resp. $r$ ) if $v$ is a vertex of $l$-type (resp. $r$-type), refer to [3] for details.

Suppose $V(P)$ consists of $n$ points $v_{1}, v_{2}, \ldots, v_{n}$. A maximal subsequence (in the sense of cyclical order) of $2 n$ vertices $v_{1}^{ \pm}, v_{2}^{ \pm}, \ldots, v_{n}^{ \pm}$on the E-cycle each of which has the same sign $+($ resp. - ) is called a positive block (resp. negative block) of $\Delta=(G, f, P ; e)$. The block number of $\Delta$ is defined to be the number of positive blocks, see [3], again, for details.

EXAMPLE 2.4. The block number of the DS-diagram $\Delta$ shown in Figure 2 is 2 . In fact, the arrangement of the diagram is $v_{1}^{+} v_{2}^{-} v_{2}^{+} v_{1}^{-}$and each of the two positive blocks consists of one vertex $\left\{v_{1}^{+}\right\}$or $\left\{v_{2}^{+}\right\}$.

\section{The structure of a Heegaard splitting derived from a flow-spine}

Given a DS-diagram $\Delta$ with E-cycle of block number $n$ of a closed oriented 3-manifold $M=M(\Delta)$, the Heegaard splitting of the manifold $M$ corresponding to the diagram $\Delta$ can be obtained as follows. Decompose the manifold $M(\Delta)$ into 4 pieces: The thickened disk $V_{3}=D^{2} \times[0,1]$ in $B$ such that $V_{3} \cap \Sigma_{+}=D^{2} \times\{1\}$, where $D^{2} \times\{1\} \subset \Sigma_{+}$contains every vertex on $\Sigma_{+}$, the collar neighborhood $V_{2}$ in $B$ of $E(G) \cap\left(\operatorname{Int} \Sigma_{+} \backslash D\right)$, the closure $V_{1}$ of the complement of $V_{2} \cup V_{3}$ in the collar $\Sigma_{+} \times[0,1]$ and the closure $V_{0}$ of $M(\Delta) \backslash\left(V_{1} \cup V_{2} \cup V_{3}\right)$. 


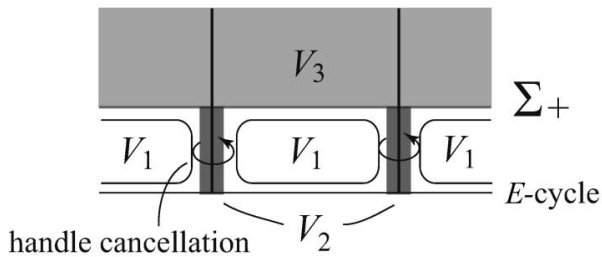

(i)

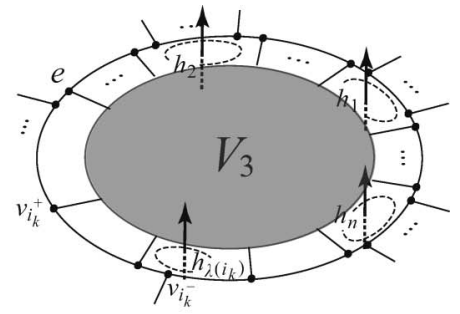

(ii)

FIGURE 4. The handle cancellation.

Set $U_{1}=U_{1}(\Delta)=V_{0} \cup V_{1}$ and $U_{2}=U_{2}(\Delta)=V_{2} \cup V_{3}$. By the above definition, $U_{1}$ and $U_{2}$ naturally become handlebodies and $U_{1} \cap U_{2}=\partial U_{1}=\partial U_{2}$. This means that $\left(U_{1}, U_{2}\right)$ gives a Heegaard splitting of the manifold $M(\Delta)$. More precisely, the above decomposition defines the handle decomposition of $M(\Delta)$ such that the union of its $i$-handles is $V_{i}$. This Heegaard splitting induces the Heegaard splitting $\left(\mathbf{U}_{1}, \mathbf{U}_{2}\right)$ of $M$ of genus $n$ by handle cancellations ( $c f$. [3]), see Figure 4 (i). (These handle cancellations play an important role in the next section.)

\section{Presentations of the fundamental group coherent to the $\operatorname{Spin}^{c} \operatorname{structure}^{-}$}

In this section, we introduce two methods for extracting a presentation of the fundamental group of the realized manifold $M(\Delta)$ of a DS-diagram $\Delta=(G, \phi, P ; e)$ with E-cycle.

4.1. A method using Heegaard splittings. Consider the Heegaard splitting $\left(U_{1}, U_{2}\right)$ of $M(\Delta)$ obtained in the above section.

Let us take the 1-handles $h_{1}, h_{2}, \ldots, h_{n}$ from the handlebody $U_{1}$ as in Figure 4 (ii) and the remaining 1-handles $h_{i_{1}}, h_{i_{2}}, \ldots, h_{i_{n_{i}}}(1 \leq i \leq n)$ as in Figure 5. The figure also shows the suffixes of vertices. Recall that the vertices are classified into two types: $l$-type and $r$-type. Focus on a vertex $v_{i_{k}}^{+}\left(1 \leq k \leq n_{i}-1\right)$. The neighborhood of the vertex $v_{i_{k}}$ in the branched surface $P$ are shown in Figure 6. In this figure, there is undefined suffix $\lambda\left(i_{k}\right) \in 1,2, \ldots, n$. We can explicitly decide this suffix of the handles on the DS-diagram as shown in Figure 4 (ii).

For the other vertices $v_{i}(1 \leq i \leq n)$ and $v_{n_{i}}(1 \leq i \leq n)$, we also drew their neighborhood in Figure 6 by checking the branching structures from the DS-diagram.

Now let us calculate the fundamental group using the Heegaard splitting $\left(U_{1}, U_{2}\right)$. Each 1-handle of $U_{1}$ determines the generator of the fundamental group. Let the generators $x_{i}(1 \leq$ $i \leq n)$ and $x_{i_{k}}\left(1 \leq i \leq n, 1 \leq k \leq n_{i}\right)$ of $\pi_{1}(M)$ correspond to the handles $h_{i}(1 \leq i \leq n)$ and $h_{i_{k}}\left(1 \leq i \leq n, 1 \leq k \leq n_{i}\right)$, respectively, with the shown orientation.

LEMMA 4.1. The relator system of the fundamental group $\pi_{1}(M)$ corresponding to the above generator system consists of the elements $r_{i}, r_{i_{k}}\left(1 \leq i \leq n, 1 \leq i \leq n_{i}\right)$ shown below: 


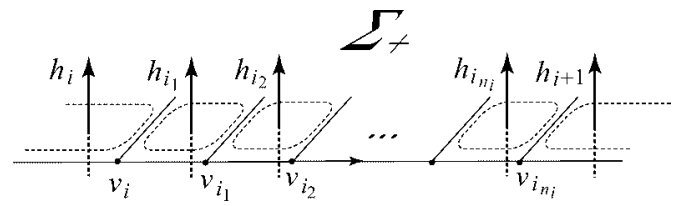

FIGURE 5. The handles $h_{i_{1}}, h_{i_{2}}, \ldots, h_{i_{n_{i}}}$.
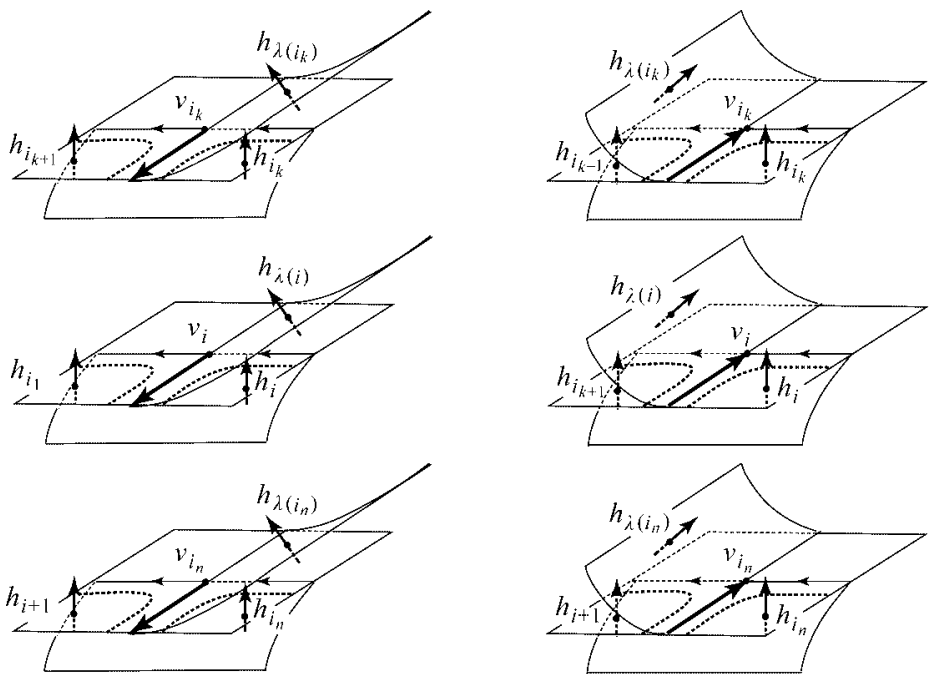

l-type

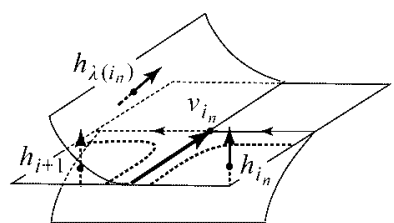

$r$-type

FIGURE 6. The neighborhood of the vertices $v_{i_{k}}, v_{i}$ and $v_{i_{n_{i}}}$.

1. If $v_{i}$ is l-type (resp. r-type), $r_{i}=x_{i_{1}} x_{\lambda(i)}^{-1} x_{i}^{-1}$ (resp. $\left.r_{i}=x_{i_{1}} x_{\lambda(i)} x_{i}^{-1}\right)$, that is, $x_{i_{1}}=x_{i} x_{\lambda(i)}\left(\operatorname{resp} . x_{i_{1}}=x_{i} x_{\lambda(i)}^{-1}\right)$.

2. If $v_{i_{k}}\left(1 \leq k \leq n_{i}-1\right)$ is l-type (resp. $r$-type), then $r_{i_{k}}=x_{i_{k+1}} x_{\lambda\left(i_{k}\right)}^{-1} x_{i_{k}}^{-1}$ (resp. $\left.r_{i_{k}}=x_{i_{k+1}} x_{\lambda\left(i_{k}\right)} x_{i_{k}}^{-1}\right)$, that is, $x_{i_{k+1}}=x_{i_{k}} x_{\lambda\left(i_{k}\right)}$ (resp. $\left.x_{i_{k+1}}=x_{i_{k}} x_{\lambda\left(i_{k}\right)}^{-1}\right)$.

3. If $v_{i_{n}}$ is l-type (resp. $r$-type), then $r_{i_{n}}=x_{i+1} x_{\lambda\left(i_{n}\right)}^{-1} x_{i_{n}}^{-1}\left(\right.$ resp. $\left.r_{i_{n}}=x_{i+1} x_{\lambda\left(i_{n}\right)} x_{i_{n}}^{-1}\right)$, that is, $x_{i+1}=x_{i_{n}} x_{\lambda\left(i_{n}\right)}\left(\right.$ resp. $\left.x_{i+1}=x_{i_{n}} x_{\lambda\left(i_{n}\right)}^{-1}\right)$.

PROOF. We can regard $V_{2} \subset U_{2}$ as 2-handles, and by Seifert van-Kampen Theorem, it is clear that the attaching slope on $U_{1}$ of each of these 2-handles defines a relator. These attaching slope are drawn in Figure 7. Now, the proposition is clear by Figure 6. 
PROPOSITION 4.2. The presentation of $\pi_{1}(M)$ with respect to the Heegaard splitting $\left(\mathbf{U}_{1}, \mathbf{U}_{2}\right)$ has the presentation $\left\langle x_{1}, x_{2}, \ldots, x_{n} \mid R_{1}, R_{2}, \ldots, R_{n}\right\rangle$, here

$$
R_{i}=x_{i} x_{\lambda(i)}^{\varepsilon(i)} x_{\lambda\left(i_{1}\right)}^{\varepsilon\left(i_{1}\right)} x_{\lambda\left(i_{2}\right)}^{\varepsilon\left(i_{2}\right)} \cdots x_{\lambda\left(i_{n}\right)}^{\varepsilon\left(i_{n}\right)} x_{i+1}^{-1}(1 \leq i \leq n),
$$

provided $\varepsilon(j)=1$ (resp. -1$)$ if $v_{j}$ is l-type (resp. r-type).

PROOF. This proposition is directly derived from Lemma 4.1 with the handle cancellations. Focus on the relator $r_{i}$ in Lemma 4.1. Since the relator $r_{i}$ has the form $x_{i_{1}}=x_{i} x_{\lambda(i)}^{\varepsilon(i)}$, where $\varepsilon(i)=1$ (resp. -1$)$ if $v_{i}$ is $l$-type (resp. $r$-type), we can present the element $x_{i_{1}}$ using elements in $\left\{x_{i} \mid 1 \leq i \leq n\right\}$. Using the relator $r_{i_{k}}$ in the same way, we can present the element $x_{i_{k+1}}$ by elements in $\left\{x_{i}, x_{i_{j}} \mid 1 \leq i \leq n, 1 \leq j \leq k\right\}$. Therefore we get by the inductive argument that all of the generators $x_{i_{j}}\left(1 \leq i \leq n, 1 \leq i \leq n_{i}\right)$ are presented by using $x_{i}(1 \leq i \leq n)$. By regarding the above relators $r_{i}, r_{i_{1}}, r_{i_{2}}, \ldots, r_{i_{i_{n}}}$ as a recurrence formula, we obtain the relator $R_{i}$.

REMARK 4.3. Each elimination of generators $x_{i_{j}}\left(1 \leq j \leq n_{i}\right)$ by the recurrence formula in the above proof corresponds to the handle cancellation of the Heegaard splitting $\left(U_{1}, U_{2}\right)$. The attaching slope of the 2-handle of $\left(\mathbf{U}_{1}, \mathbf{U}_{2}\right)$ is drawn in Figure 8.

REMARK 4.4. The above method has no ambiguity of cyclic conjugations of the relators $R_{1}, \ldots, R_{n}$, not as in the case where we use Heegaard diagrams only. In fact, we specified the start points of the attaching slopes to read the words of relators in the above argument. This rigidity comes from the point that we are considering not only the 3-manifold $M$ but the $\operatorname{Spin}^{c}$ structure $[\mathcal{V}(\Delta)]$, and the presentation are compatible with $[\mathcal{V}(\Delta)]$. In other

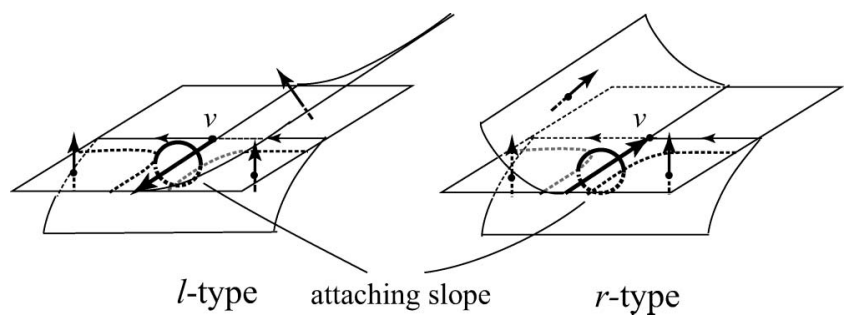

FIGURE 7. The attaching slope of a 2-handle of $\left(U_{1}, U_{2}\right)$.

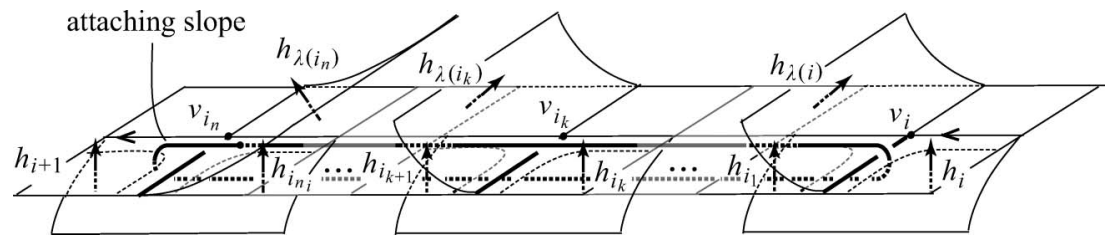

FIGURE 8. An attaching slope of a 2-handle of $\left(\mathbf{U}_{1}, \mathbf{U}_{2}\right)$. 

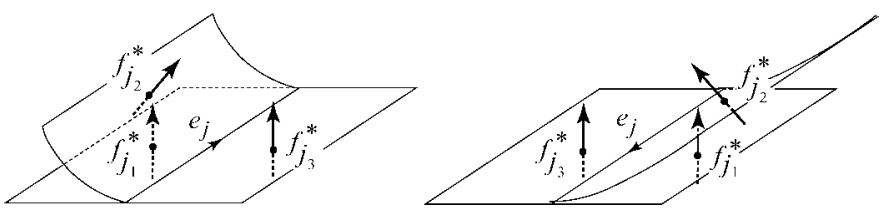

FIGURE 9. The suffixes of faces around the edge $e_{j}$.

words, the above presentation is associated to the $\operatorname{Spin}^{c}$ structure $[\mathcal{V}(\Delta)]$. This rigidity plays an important role in the Section 7.

4.2. A method using every face In this section, all branched simple spines are assumed to be standard ( $c f$. Section 2.1) for simplicity.

Recall that the structure of $V(P) \subset S(P) \subset P \subset M$ induces a CW-decomposition $X=X(\Delta)$ of the manifold $M=M(\Delta)$. Set $V(P)=\left\{v_{1}, v_{2}, \ldots, v_{k}\right\}, S(P) \backslash V(P)=$ $\left\{e_{1}, e_{2}, \ldots, e_{l}\right\}$ and $P \backslash S(P)=\left\{f_{1}, f_{2}, \ldots, f_{m}\right\}$. Each face $f_{i}$ of $P$ corresponds to the two faces $f_{i}^{+} \subset \Sigma_{+}$and $f_{i}^{-} \subset \Sigma_{-}$on $\Delta$. Let $X^{*}$ denote the dual complex of $X$ and for each $i$-cell $c$ of $X$, the corresponding $(3-i)$-cell be denoted as $c^{*} . X^{*}$ has only one vertex, thus the set of edges $f_{i}^{*}$ directly represent the generator system $S$ for the fundamental group $\pi_{1}(M)$. Orient the edge $f_{i}^{*}$ to intersect with $f_{i}$ from the side of $\Sigma_{+}$to $\Sigma_{-}$.

Let $\left[f_{i}^{*}\right]$ denote the generator of the fundamental group $\pi_{1}(M)$ represented by the oriented loop $f_{i}^{*}$. For each edge $e_{j} \in E(P)$, there are three faces $f_{j_{1}}, f_{j_{2}}, f_{j_{3}} \in F(P)$ (possibly contain the multiplicity) whose boundaries contain this edge. (Recall the definition of a fake surface.) There are two cases of the branching structure around the edge $e_{j}$. We adopt the suffixes shown in Figure 9. The edge $e$ corresponds to a 2-cell $e^{*}$ of $X^{*}$, hence $\left[f_{j_{1}}^{*}\right]\left[f_{j_{2}}^{*}\right]\left[f_{j_{3}}^{*}\right]^{-1}=1 \in \pi_{1}(M)$.

LEMMA 4.5 (Ishii [5]). In this way, we get a presentation of the fundamental group $\pi_{1}(M)$ with $m$ generators and $l$ relators.

$$
\left\langle\left[f_{i}^{*}\right](i=1,2, \ldots, m) \mid\left[f_{j_{1}}^{*}\right]\left[f_{j_{2}}^{*}\right]\left[f_{j_{3}}^{*}\right]^{-1}(j=1,2, \ldots, l)\right\rangle .
$$

Proof. The union $X^{*(0)} \cup X^{*(1)}$ of 0 -skeleton $X^{*(0)}$ and 1-skeleton $X^{*(1)}$ of $X^{*}$ is a bouquet with $m$ loops, and whose fundamental group is a free group $\left\langle f_{1}^{*}, f_{2}^{*}, \ldots, f_{m}^{*} \mid-\right\rangle$. Then by applying the Seifert van-Kampen Theorem to every 2-cell attaching of each 2-cell $e_{i}^{*}$, we obtain the above presentation of the manifold $M(\Delta)$. 


\section{Diagrams of the maximal abelian covering spaces}

Given a DS-diagram $\Delta$ with E-cycle, set $M=M(\Delta)$. We use the same notation as in Section 3, 4. Consider the Cayley graph $\Gamma$ of the homology group $H_{1}(M)$ with respect to the generating system represented by $\left\{f_{i}^{*} \mid i=1,2, \ldots, n\right\}$. Take a copy of $\Delta$ for each vertex of the graph $\Gamma$. The copy of the diagram $\Delta$ corresponding to the vertex $g$ of $\Gamma$ is denoted by $\Delta_{g}=\left(G_{g}, \phi_{g}, P_{g}\right)$, and the cells of $X\left(\Delta_{g}\right)$ corresponding to $\Delta_{g}$ are denoted by $V\left(P_{g}\right)=$ $\left\{v_{1}^{g}, v_{2}^{g}, \ldots, v_{k}^{g}\right\}, S\left(P_{g}\right) \backslash V\left(P_{g}\right)=\left\{e_{1}^{g}, e_{2}^{g}, \ldots, e_{l}^{g}\right\}, P \backslash S\left(P_{g}\right)=\left\{f_{1}^{g}, f_{2}^{g}, \ldots, f_{m}^{g}\right\}$ and $B_{g}$. Let $e_{g}$ be the E-cycle of $\Delta$ and set $\partial B_{g} \backslash e_{g}=\Sigma_{+}^{g} \cup \Sigma_{-}^{g}$. Set $v_{i}^{ \pm g}=\phi_{g}^{-1}\left(v_{i}\right) \cap \Sigma_{ \pm}$, $e_{i}^{ \pm g}=\phi_{g}^{-1}\left(e_{i}\right) \cap \Sigma_{ \pm}$and $f_{i}^{ \pm g}=\phi_{g}^{-1}\left(f_{i}\right) \cap \Sigma_{ \pm}$. Let $\iota_{g h}$ denote the natural identification map from $\partial B_{g}$ to $\partial B_{h}$.

Now, let us construct the maximal abelian covering space $\hat{M}$ of the manifold $M$. The faces $f_{i}^{+g}$ and $f_{i}^{-h}$ are identified by $\phi_{h} \circ \iota_{g h}$ if and only if the vertices $g$ and $h$ of the graph $\Gamma$ are connected by an edge. Let $\hat{\phi}$ denote this identification.

Proposition 5.1. The maximal abelian covering space $\hat{M}$ is obtained by the following formula:

$$
\hat{M}=\left(\bigcup_{g \in H_{1}(M)} B_{g}\right) / \hat{\phi}
$$

\section{The spiders associated to Smooth $\operatorname{Spin}^{c}$ structures}

Consider a DS-diagram $\Delta=(G, \phi, P ; e)$ with an E-cycle. In this section, we give a brief survey of Benedetti-Petronio's method for constructing a spider associated to the (homotopy class of) vector field(s) $\langle\mathcal{V}\rangle=\langle\mathcal{V}(\Delta)\rangle$ on the realized manifold $M=M(\Delta)$ in our terminology. Assume that $P$ is a standard spine.

Consider the dual complex $X^{*}$ defined in Section 4.2. Recall that it consists of a 0-cell $\left\{B^{*}\right\}, 1$-cells $\left\{f_{1}^{*}, \ldots, f_{m}^{*}\right\}, 2$-cells $\left\{e_{1}^{*}, \ldots, e_{l}^{*}\right\}$ and 3 -cells $\left\{v_{1}^{*}, \ldots, v_{k}^{*}\right\}$ and that this is a one-vertex triangulation of $M$. Choose the barycenter $\sigma_{c_{q}^{r}}$ for each $r$-cell $c_{q}^{r}$ of $X^{*}$. It may be assumed to lie on the spine $P$. Consider a spider consisting of arcs connecting $\sigma$ to the barycenter of each cell, whose inner points do not intersect with $P$. We assume that each edge connects to $\sigma_{c_{q}^{r}}$ from the one side of $P$ corresponding to $\Sigma_{+}$. The directions of edges are determined by the dimension $r$ of $c_{q}^{r}$ as introduced in Section 1.2. Denote this spider by $s^{X^{*}}$. In [2], Benedetti and Petronio determined the inverse map of Turaev's canonical map $\Phi: \operatorname{Eul}(M) \rightarrow \operatorname{Vect}(M)$, recall Theorem 1.1. In our terminology, the result can be restated as follows.

THEOREM 6.1 (Benedetti-Petronio [2])). In the above setting, we have $\left[\mathrm{s}^{X^{*}}\right]=$ $\Phi^{-1}([\mathcal{V}])$. 


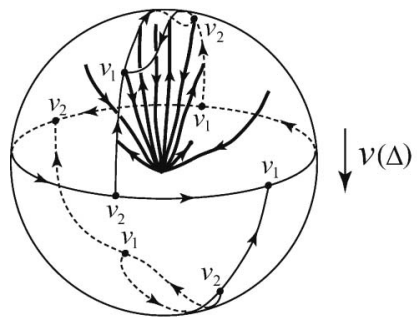

(i)

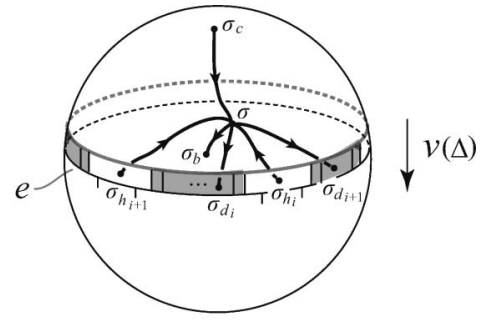

(ii)

FIgURE 10. The spiders $s^{X^{*}}$ and $s$.

EXAMPLE 6.2. Let $\Delta$ be a DS-diagram with E-cycle of lens space $L(3,1)$ shown in Figure 10 (i). The spider $s^{X^{*}}$ associated to the vector field $\mathcal{V}(\Delta)$ is drawn in this figure.

\section{A formula for the Reidemeister-Turaev torsion}

In this section, we use the same notation as in Section 3-6.

Let $\Delta$ be a DS-diagram of block number $n$ and set $M=M(\Delta)$. The branched spine $P$ is assumed to be standard. As we have seen above, the diagram naturally induces the Heegaard splitting $\left(\mathbf{U}_{1}, \mathbf{U}_{2}\right)$ of genus $n$. This Heegaard splitting induces the cellular structure $\mathcal{C}$ which consists of one 0 -cell $\mathcal{C}_{0}=\{b\}, n$ 1-cells $\mathcal{C}_{1}=\left\{h_{1}, \ldots, h_{n}\right\}, n$ 2-cells $\mathcal{C}_{2}=\left\{d_{1}, \ldots, d_{n}\right\}$ and one 3-cell $\mathcal{C}_{3}=\{c\}$. Regard $C_{*}(\hat{\mathcal{C}})=C_{*}(\hat{\mathcal{C}} ; \mathbf{Z})$ as a $\mathbf{Z}\left[H_{1}(M)\right]$-module.

Choose the presentation $\left\langle x_{1}, \ldots, x_{n} \mid R_{1}, \ldots, R_{n}\right\rangle$ of the fundamental group $\pi_{1}(M)$ obtained in Proposition 4.2. Let $\varphi: \mathbf{Z}\left[H_{1}(M)\right] \rightarrow F^{\times}$be a ring homomorphism with $t_{i}=\varphi\left(x_{i}\right)$. Then we can define the based twisted chain complex $C_{*}^{\varphi}$

$$
\left(0 \rightarrow F \otimes_{\varphi} C_{3}(\hat{\mathcal{C}}) \stackrel{\partial_{2}^{\varphi}}{\longrightarrow} F \otimes_{\varphi} C_{2}(\hat{\mathcal{C}}) \stackrel{\partial_{1}^{\varphi}}{\rightarrow} F \otimes_{\varphi} C_{1}(\hat{\mathcal{C}}) \stackrel{\partial_{0}^{\varphi}}{\longrightarrow} F \otimes_{\varphi} C_{0}(\hat{\mathcal{C}}) \rightarrow 0\right)
$$

Our aim in this section is to find the basis of this chain complex, i.e. the fundamental family of cells of $\hat{\mathcal{C}}$ which corresponds to the smooth $\operatorname{Spin}^{c}$ structure $[\mathcal{V}(\Delta)]$.

Consider the diagram $\left\{\Delta_{g}\right\}_{g \in H_{1}(M)}$ of the maximal abelian covering space $\hat{M}$ constructed in Section 5. Choose the fundamental family of cells of $\hat{\mathcal{C}}$ as $C_{0}^{\varphi}(\hat{\mathcal{C}})=\langle\hat{b}\rangle$, $C_{1}^{\varphi}(\hat{\mathcal{C}})=\left\langle\hat{h}_{1}, \ldots, \hat{h}_{n}\right\rangle, C_{2}^{\varphi}(\hat{\mathcal{C}})=\left\langle\hat{d}_{1}, \ldots, \hat{d}_{n}\right\rangle$ and $C_{3}^{\varphi}(\hat{\mathcal{C}})=\langle\hat{c}\rangle$, where each lift corresponds to $\Delta_{1}$.

Take a base point $\sigma \in M$ not lying on the spine $P$, and barycenters $\sigma_{b} \in b, \sigma_{h_{j}} \in h_{j}$, $\sigma_{d_{i}} \in d_{i}, \sigma_{c} \in c$ for each cells of $\mathcal{C}$.

THEOREM 7.1. A combinatorial Spin ${ }^{c}$ structure $\left[s^{c}\right]$ for $X$ represented by the spider ${ }^{\mathcal{C}}$ drawn in Figure 10 (ii) corresponds to the smooth $\operatorname{Spin}^{c}$ structure [V] by the $H_{1}(M)$ equivariant bijection $\Phi$ introduced in Section 1.2. 
ProOF. We will give the $H_{1}(M)$-equivariant bijection directly by constructing a common subdivision. Consider the $\mathrm{CW}$-structure $\mathcal{C}^{\prime}$ induced from the Heegaard splitting $\left(U_{1}, U_{2}\right)$ which consists of a 0 -cell $X_{0}=\{b\}, 1$-cells $X_{1}=\left\{h_{i} \mid 1 \leq i \leq n\right\} \cup \bigcup_{1 \leq i \leq n}\left\{h_{i_{k}} \mid 1 \leq k \leq n_{i}\right\}$, 2-cells $X_{2}=\left\{d_{i} \mid 1 \leq i \leq n\right\} \cup \bigcup_{1 \leq i \leq n}\left\{d_{i_{k}} \mid 1 \leq k \leq n_{i}\right\}$ and a 3-cell $X_{3}=\{c\}$.

Claim 7.2. $\mathcal{C}^{\prime}$ is a subdivision of $\mathcal{C}$.

This is clear from their definitions. In fact, we can easily find that $h_{i_{k}} \subset d_{i}$ and $d_{i_{k}} \subset d_{i}$ for each $1 \leq i \leq n$.

CLAIM 7.3. There exists a common subdivisional cellular decomposition $Z$ of the two complexes $X^{*}$ and $\mathcal{C}$, whose unique 0 -cell is $b$.

Proof of Claim 7.3. By Claim 7.2, it is sufficient to prove that there exists a common subdivisional cellular decomposition of the two complexes $X^{*}$ and $\mathcal{C}^{\prime}$, whose unique 0 -cell is $b$. Focus on the upper-half part of the DS-diagram. Add edges and vertices to the polyhedron $P$ drawn on the diagram along the circle $\partial\left(V_{3} \cup P\right)$ and denote the resulting polyhedron by $P^{\prime}$, see Figure 11 .

The polyhedron $P^{\prime}$ also induces the cellular decomposition $Y$ of $M$, thus we can consider its dual decomposition $Y^{*}$. Note that $X^{*}$ and $Y^{*}$ has the natural common subdivision $Z$ such that their 2-skeletons $Z^{(2)},\left(X^{*}\right)^{(2)}$ and $\left(Y^{*}\right)^{(2)}$ satisfies $\left|Z^{(2)}\right|=\left|\left(X^{*}\right)^{(2)}\right| \cup\left|\left(Y^{*}\right)^{(2)}\right|$ and that the unique 0 -cell of $Z$ is $b$. By the above construction, the cells of $\mathcal{C}^{\prime}$ and $Y^{*}$ have the following properties:

1. $h_{i}=\left(f_{i}^{\prime}\right)^{*}, h_{i_{k}}=\left(f_{i_{k}}^{\prime}\right)^{*}\left(1 \leq i \leq n, 1 \leq k \leq i_{n}\right)$, where $f_{i}^{\prime}$ is the face of $P^{\prime}$ corresponding to the handle $h_{i}$, and $f_{i_{k}}^{\prime}$ is the face corresponding to the handle $h_{i_{k}}$,

2. $d_{i}=\left(e_{i}^{\prime}\right)^{*}, d_{i_{k}}=\left(e_{i_{k}}^{\prime}\right)^{*}\left(1 \leq i \leq n, 1 \leq k \leq i_{n}\right)$, where $e_{i}^{\prime}$ is the edge of $P^{\prime}$ corresponding to the 2-handle $d_{i}$, and $e_{i_{k}}^{\prime}$ is the edge corresponding to the 2-handle $d_{i_{k}}$,

3. Every $p$-cell $(1 \leq p \leq 3)$ of $Y^{*}$ except for $h_{i} h_{i_{k}} d_{i}, d_{i_{k}}\left(1 \leq i \leq n, 1 \leq k \leq i_{n}\right)$ is contained in a 3-cell $d$.

This means that $Y^{*}$ is a subdivision of $\mathcal{C}^{\prime}$. This follows that $Z$ is a subdivision of $\mathcal{C}^{\prime}$.

Let us continue the proof of Theorem 7.1. We denote by $s^{X^{*}}$ the spider associated to [V] constructed in Section 6. Take the spiders $s^{\mathcal{C}^{\prime}}$ and $s^{Z}$ for $\mathcal{C}^{\prime}$ and $Z$, respectively, in the same

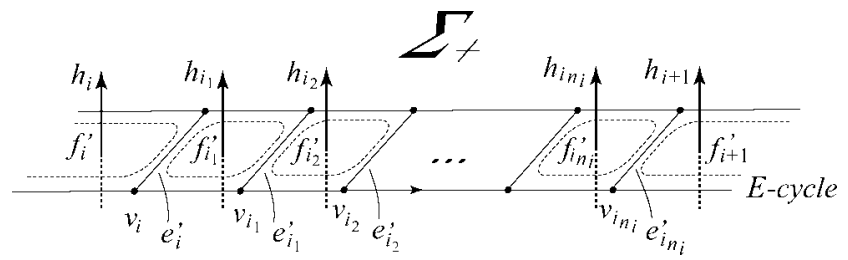

FIGURE 11. The polyhedron $P^{\prime}$. 
way as $s^{X^{*}}$. Choose a lift $\hat{\sigma}$ of the base point $\sigma$ on Int $B_{0} \subset \hat{M}$. Let $\hat{s}^{X}$ denote a lift of $s^{X}$ based on $\hat{\sigma}$. We denote by $\hat{s}^{\mathcal{C}}, \hat{s}^{\mathcal{C}^{\prime}}$ and $\hat{s}^{Z}$ the lifts of $s^{\mathcal{C}}, s^{\mathcal{C}^{\prime}}$ and $s^{Z}$, respectively, chosen in the same way. Recall that these lifts define the fundamental family of cells $S^{X}, S^{\mathcal{C}}, S^{\mathcal{C}^{\prime}}$ and $S^{Z}$.

CLAIM 7.4. $\quad\left[s^{Z}\right]=\Psi_{X^{*} Z}\left(\left[s^{X^{*}}\right]\right)$.

Proof of Claim 7.4. Choose a cell $a \in X^{*}$ and a cell $a^{\prime} \in Z$ with $\left|a^{\prime}\right| \subset|a|$. By the definition of the map $\Psi_{X^{*} Z}$, it is sufficient to prove $\left|\hat{a}^{\prime}\right| \subset|\hat{a}|$, where $\hat{a} \in S^{X^{*}}$ and $\hat{a}^{\prime} \in S^{Z}$ are lifts of $a$ and $a^{\prime}$, respectively. The path $p_{a^{\prime}}$ of $s^{\prime}$ connecting $\sigma$ to $\sigma_{a^{\prime}}$ can be homotopically deformed to the composition of the path $p_{a}$ of $s$ connecting $\sigma$ to $\sigma_{a}$ and the path $p_{a a^{\prime}}$ from $\sigma_{a}$ to $\sigma_{a^{\prime}}$ in $|a|$. This deformation is supported in the 3-ball $B$. Since the lift $\hat{p}_{a a^{\prime}}$ of $p_{a a^{\prime}}$ contains $\hat{\sigma}_{a}$ as an endpoint lying in $\hat{a}$, we get that $\left|\sigma_{a^{\prime}}\right| \subset\left|\sigma_{a}\right|$.

CLAim 7.5. $\left[s^{Z}\right]=\Psi_{\mathcal{C Z}}\left[s^{\mathcal{C}}\right]$.

The proof of this claim is the same as Claim 7.4.

By the above claims, we get $\left[s^{\mathcal{C}}\right] \stackrel{\Psi_{\mathcal{C Z}}}{\longrightarrow}\left[s^{Z}\right] \stackrel{\Psi_{X^{*} Z}^{-1}}{\longrightarrow}\left[s^{X^{*}}\right] \stackrel{\Phi}{\longrightarrow}[\mathcal{V}]$, This completes the proof of Theorem 7.1.

COROLLARY 7.6. The fundamental family of cells $\left\{\hat{b}, \hat{h}_{1}, \ldots, \hat{h}_{n}, \hat{d}_{1}, \ldots, \hat{d}_{n}, \hat{c}\right\}$ of $\hat{X}$ induces the combinatorial Spin ${ }^{c}$ structure $[s]$.

Proof. Choose a lift of the base point $\sigma$ on $\operatorname{Int} B_{0} \subset \hat{M}$. Then the assertion is clear from Theorem 7.1 and the construction of the maximal abelian covering explained in Section 5.

Due to the above corollary, it is sufficient to determine the presentation matrices of the boundary operators of the twisted chain complex $C^{\varphi}(\mathcal{C})$ with respect to the basis $\left\{\hat{b}, \hat{h}_{1}, \ldots, \hat{h}_{n}, \hat{d}_{1}, \ldots, \hat{d}_{n}, \hat{c}\right\}$ to compute the Reidemeister-Turaev torsion of the pair $(M,[\mathcal{V}])$.

THEOREM 7.7. The fundamental family of cells $\left\{\hat{b}, \hat{h}_{1}, \ldots, \hat{h}_{n}, \hat{d}_{1}, \ldots, \hat{d}_{n}, \hat{c}\right\}$ in the maximal abelian covering space $\hat{M}$ induces the following presentation matrices of the boundary operators $\partial_{i}^{\varphi}(0 \leq i \leq 2)$ :

$$
\partial_{0}^{\varphi}=\left(\begin{array}{lll}
t_{1}-1 & \cdots & t_{n}-1
\end{array}\right), \partial_{1}^{\varphi}=\left(\varphi \circ \operatorname{proj}\left(\frac{\partial R_{i}}{\partial x_{j}}\right)\right)_{1 \leq i, j \leq n}, \partial_{2}^{\varphi}=\left(\begin{array}{c}
\varphi\left(g_{1}\right)-1 \\
\vdots \\
\varphi\left(g_{n}\right)-1
\end{array}\right),
$$

where $\frac{\partial}{\partial x_{j}}$ denotes the Fox's free differential calculus, proj : $\pi_{1}(M) \rightarrow H_{1}(M)$ denotes the canonical projection and the element $g_{j} \in H_{1}(M)$ is represented by the dual loop $f_{i_{j}}^{*}$ of the face $f_{i_{j}}$ of the spine $P$ shown in Figure 12. (In the figure, $m_{i}$ denotes the meridian of the 1-handle $h_{i}$ for each $1 \leq i \leq n$.) 


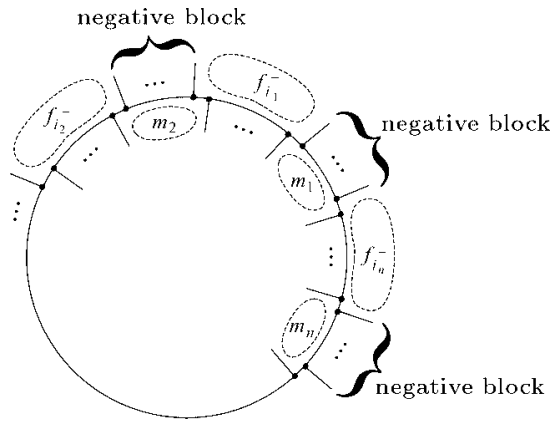

FIGURE 12. The faces $f_{i_{j}}$.

PROOF. For simplicity, we determine the presentation matrices for boundary operators of the untwisted chain complex over $\mathbf{Z}\left[H_{1}(M)\right]$

$$
\left(0 \rightarrow C_{3}(\hat{\mathcal{C}}) \stackrel{\partial_{2}}{\rightarrow} C_{2}(\hat{\mathcal{C}}) \stackrel{\partial_{1}}{\rightarrow} C_{1}(\hat{\mathcal{C}}) \stackrel{\partial_{0}}{\rightarrow} C_{0}(\hat{\mathcal{C}}) \rightarrow 0\right)
$$

The determination of $\partial_{0}$ is easy. In fact, each (oriented) 1 -cell $h_{j}$ corresponds to the generator $x_{j}$ and the two boundary points of $\hat{h}_{j}$ are $x_{j} \cdot \hat{b}$ and $-1 \cdot \hat{b}$. This means

$$
\partial_{0}\left(\hat{h}_{j}\right)=\left(x_{j}-1\right) \cdot \hat{b} \text {. }
$$

By the construction of the relators $R_{i}$, their words are read along the boundary of the lifts $\hat{d}_{i}$ in the maximal abelian covering space $\hat{M}$ starting from the 0 -cell $\hat{b}$. Thus it is clear from the definition of Fox's free differential calculus that the coefficient of each base $\hat{h}_{j}$ for the image of $\hat{d}_{i}$ by the boundary operator $\partial_{1}$ becomes $\operatorname{proj}\left(\frac{\partial R_{i}}{\partial x_{j}}\right)$ itself. That is, we get the formula $\partial_{1}\left(\hat{d}_{i}\right)=\sum_{j=1}^{n} \operatorname{proj}\left(\frac{\partial R_{i}}{\partial x_{j}}\right) \cdot \hat{h}_{j}$, and obtain the explicit presentation of $\partial_{1}$.

Now, let us focus on the boundary operator $\partial_{2}$. As we have seen in Section 5 , each face $f_{i j}^{+1}$ is identified with $f^{-g_{j}}$ by the identification map $\hat{f}$. This means that the positive side of 2-cell $g_{j} \cdot \hat{d}_{i}$ of $(\hat{\mathcal{C}})$ is attached to the 3 -cell $\hat{c}$, see Figure 13 . Then we get the presentation matrix $\left(g_{1}-1 \cdots g_{n}-1\right)^{t}$ of the boundary operator $\partial_{2}$.

The next formula of the Reidemeister-Turaev torsion immediately follows from the above theorem.

THEOREM 7.8. Let the twisted chain complex $C_{*}^{\varphi}(M)$ be acyclic. Then there exist two indices $1 \leq k, l \leq n$ such that

$$
\tau^{\varphi}\left(M,[\mathcal{V}], \mathfrak{o}_{M}\right)=(-1)^{k+l+n-1} \operatorname{sign}\left(\tau\left(C_{*}(X ; \mathbf{R})\right)\right) \frac{\operatorname{det} B_{k, l}}{\left(t_{k}-1\right)\left(\varphi\left(g_{l}\right)-1\right)} \in F^{\times},
$$




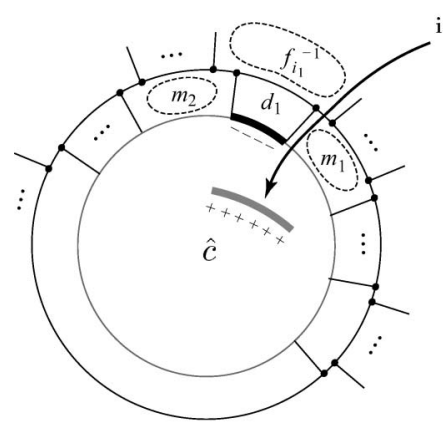

$\Delta_{1}$

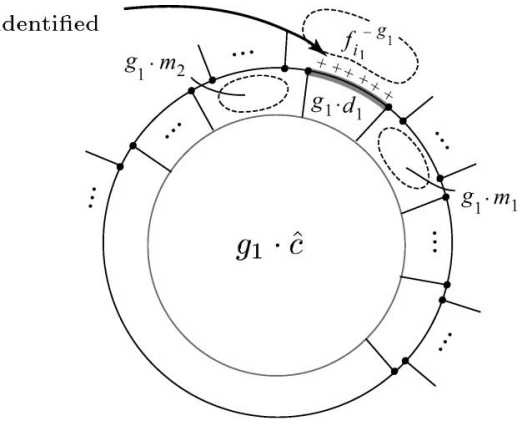

$\Delta_{g_{1}}$

FIGURE 13. The coefficient of $d_{1}$ for $\partial_{2}^{\varphi}(\hat{b})$ is $\varphi\left(g_{1}\right)-1$.

where $B_{k, l}$ is the matrix obtained by removing the $k$-th row and the $l$-th column from the matrix $\left(\varphi \circ \operatorname{proj}\left(\frac{\partial R_{i}}{\partial x_{j}}\right)\right)_{1 \leq i, j \leq n}$, and $C_{*}(X ; \mathbf{R})$ is a chain complex with ordered basis $\left\{b, h_{1}, \ldots, h_{n}, d_{1}, \ldots, d_{n}, c\right\}$ and homology orientation $\mathfrak{o}_{M}$.

Proof. Set $c_{3}=\{\hat{b}\}, c_{2}=\left\{\hat{d}_{1}, \ldots, \hat{d}_{n}\right\}, c_{1}=\left\{\hat{h}_{1}, \ldots, \hat{h}_{n}\right\}$, and $c_{0}=\{\hat{c}\}$. Since the chain complex $C_{*}^{\varphi}(M)$ is acyclic, there exist appropriate bases $b_{3}=\{\hat{b}\}, b_{2}=$ $\left\{\hat{d}_{1}, \ldots, \hat{d}_{l-1}, \hat{d}_{l+1}, \ldots, \hat{d}_{n}\right\}, b_{1}=\left\{\hat{h}_{k}\right\}, b_{0}=\emptyset$ which satisfy $\operatorname{dim} \partial_{i}\left(b_{i+1}\right)=\operatorname{dim} b_{i+1}$. Then it follows from Theorem 7.7 that

$$
\begin{aligned}
\tau^{\varphi}\left(M,[\mathcal{V}], \mathfrak{o}_{M}\right) & =\operatorname{sign}\left(\tau\left(C_{*}(X ; \mathbf{R})\right)\right) \frac{\left[\partial_{1}^{\varphi}\left(b_{2}\right) b_{1} / c_{1}\right]}{\left[\partial_{0}^{\varphi}\left(b_{1}\right) b_{0} / c_{0}\right]\left[\partial_{2}^{\varphi}\left(b_{3}\right) b_{2} / c_{2}\right]} \\
& =\operatorname{sign}\left(\tau\left(C_{*}(X ; \mathbf{R})\right)\right) \frac{(-1)^{k+n} \operatorname{det} B_{k, l}}{(-1)^{l-1}\left(t_{k}-1\right)\left(\varphi\left(g_{l}\right)-1\right)} \in F^{\times} .
\end{aligned}
$$

REMARK 7.9. Recall that the acyclicness of the twisted chain complex $C_{*}^{\varphi}(M)$ depends only on the topology of the 3-manifold $M$ and the representation $\varphi$, and the Reidemeister-Turaev torsion does not vanish if and only if the chain complex $C_{*}^{\varphi}(M)$ is acyclic. Hence the above formula can be applied universally to arbitrary pairs of 3-manifolds and $\operatorname{Spin}^{c}$ structures on them. Compare this result with Turaev's one, see Chapter VIII Theorem 2.2 in [18].

\section{Examples and applications}

8.1. Lens spaces. Let $M$ be a Seifert fibered manifold. A vector field on $M$ is said to be standard if it is everywhere tangential to a fiber. A $\operatorname{Spin}^{c}$ structure is said to be standard if it is represented by a standard vector field. 

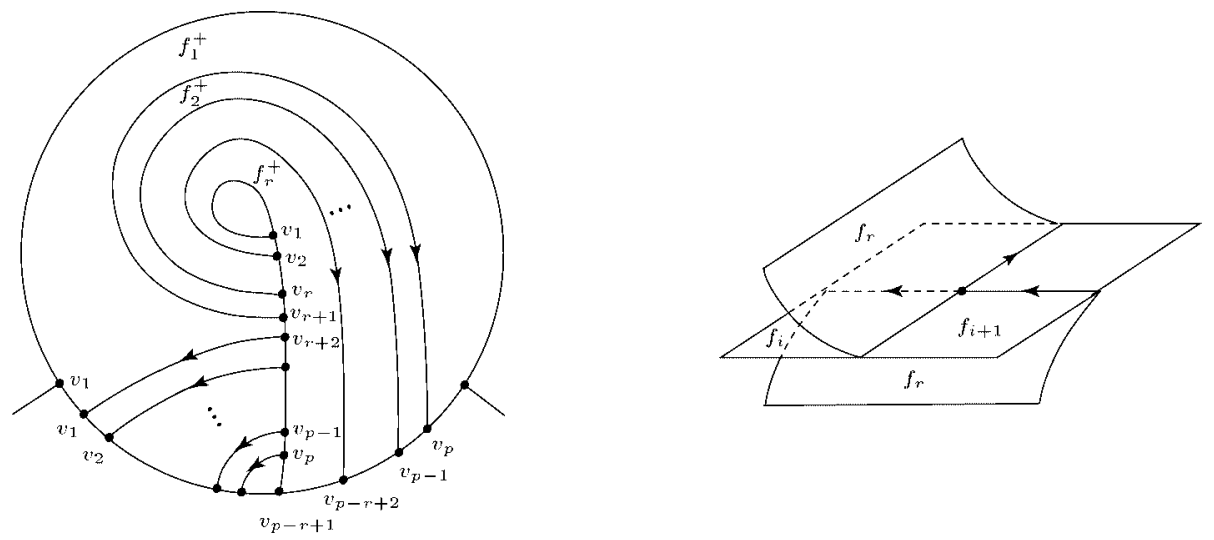

fr-

FIGURE 14. DS-diagram of $(L(p, q),[\mathcal{V}])$.

Consider the lens space $L(p, q)$ and a standard $\operatorname{Spin}^{c}$ structure $\left[\mathcal{V}_{\mathrm{st}}\right]$ on the manifold. The left-hand side of Figure 14 illustrates a DS-diagram corresponding to the pair $\left(L(p, q),\left[\mathcal{V}_{0}\right]\right)$, see [3].

Recall that $\pi_{1}(L(p, q))=H_{1}(L(p, q))=\left\langle\left[f_{1}^{*}\right] \mid\left[f_{1}^{*}\right]^{p}\right\rangle$. Each vertex $v_{i}$ of the spine $P$ is $r$-type, see the right-hand side of Figure 14 and thus we get by the argument of Proposition 4.2 that $\left[f_{r}^{*}\right]=\left[f_{1}^{*}\right]^{r}$. Let $\zeta$ be a primitive $p$-th root of unity and $\varphi: \mathbf{Z}\left[H_{1}(L(p, q))\right] \rightarrow \mathbf{Q}(\zeta)$ be the ring homomorphism with $\varphi\left(\left[f_{1}^{*}\right]\right)=\zeta$. Then we obtain the following presentation matrices of boundary operators modulo $\pm \varphi\left(H_{1}(L(p, q))\right): \partial_{2}^{\varphi}=\left(\zeta^{r}-1\right), \partial_{1}^{\varphi}=0, \partial_{0}^{\varphi}=$ $(\zeta-1)$. Then we get

$$
\tau^{\varphi}\left(L(p, q),\left[\mathcal{V}_{\mathrm{st}}\right], \mathfrak{o}_{L(p, q)}\right)=\frac{1}{(\zeta-1)\left(\zeta^{r}-1\right)} \in \mathbf{C}
$$

Remark that if we substitute $\zeta^{q}$ to $\zeta$ in $\frac{1}{(\zeta-1)\left(\zeta^{r}-1\right)}$, we get $\frac{1}{(\zeta-1)\left(\zeta^{q}-1\right)}$. The above shows that the $\operatorname{Spin}^{c}$ structure $[\mathcal{V}]$ on $L(p, 1)$, for example, which has the value $\frac{\zeta^{k}}{(\zeta-1)(\zeta-1)}$ $(0<k<p)$ is not the standard one. This observation allows us to interpret that for lens spaces the Reidemeister-Turaev torsions of standard $\operatorname{Spin}^{c}$ structures are 'standard' as values (or polynomials).

QUESTION 8.1. Can the Reidemeister-Turaev torsion of a general Seifert fibered manifold with a standard $\operatorname{Spin}^{c}$ structure be interpreted to be 'standard' as a value (or a polynomial) in any meaning as above?

8.2. How to use the Reidemeister-Turaev torsion to compare $\operatorname{Spin}^{c} \operatorname{structures}^{2}$ Consider the two manifolds $\left(M_{1},\left[\mathcal{V}_{1}\right]\right)$ and $\left(M_{2},\left[\mathcal{V}_{2}\right]\right)$ equipped with $\operatorname{Spin}^{c}$ structures shown 

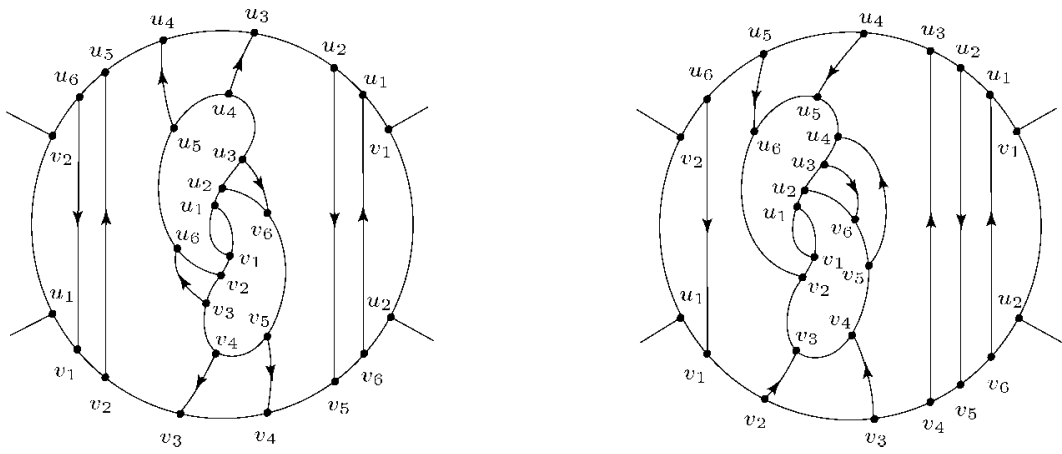

FIGURE 15. DS-diagrams of $\left(M_{1},\left[\mathcal{V}_{1}\right]\right)$ and $\left(M_{2},\left[\mathcal{V}_{2}\right]\right)$.

in Figure 15. In [7], we explain the strong relationship between DS-diagrams and Heegaard diagrams, and the argument shows that $M_{1}$ and $M_{2}$ are in fact the same smooth manifold $M$.

The presentation of $\pi_{1}\left(M_{1}\right)$ with respect to the left-hand side diagram is

$$
\left\langle x_{1}, x_{2} \mid x_{1} x_{2}^{-1} x_{1} x_{2}^{-3} x_{1} x_{2}^{-1}, x_{2} x_{1}^{-1} x_{2} x_{1}^{-3} x_{2} x_{1}^{-1}\right\rangle,
$$

and that of the right-hand side diagram is

$$
\left\langle y_{1}, y_{2} \mid y_{1} y_{2}^{-1} y_{1} y_{2}^{-1} y_{1}^{3} y_{2}^{-1}, y_{2} y_{1}^{-1} y_{2} y_{1}^{-1} y_{2}^{3} y_{1}^{-1}\right\rangle \text {. }
$$

In these presentation, the map $x_{1} \mapsto y_{2}^{-1}, x_{2} \mapsto y_{1}^{-1}$ gives an isomorphism.

Set $R_{1}=x_{1} x_{2}^{-1} x_{1} x_{2}^{-3} x_{1} x_{2}^{-1}, R_{2}=x_{2} x_{1}^{-1} x_{2} x_{1}^{-3} x_{2} x_{1}^{-1}$, and $R_{1}^{\prime}=y_{1} y_{2}^{-1} y_{1} y_{2}^{-1} y_{1}^{3} y_{2}^{-1}$, $R_{2}^{\prime}=y_{2} y_{1}^{-1} y_{2} y_{1}^{-1} y_{2}^{3} y_{1}^{-1}$. Then the first homology groups are presented as $H_{1}\left(M_{1}\right)=$ $\left\langle x_{1}, x_{2} \mid 3 x_{1}-5 x_{2},-5 x_{1}+3 x_{2}\right\rangle, H_{1}\left(M_{2}\right)=\left\langle x_{1}, x_{2} \mid-5 y_{1}+3 y_{2}, 3 y_{1}-5 y_{2}\right\rangle$.

By elementary calculation, we get from the above presentation of $H_{1}\left(M_{1}\right)$ that $o\left(x_{1}\right)=$ 16 and $x_{2}=7 x_{1}$, that is, $H_{1}\left(M_{1}\right)=\left\langle x_{1} \mid x_{1}^{16}\right\rangle=\mathbf{Z} / 16 \mathbf{Z}$. Similarly, we get a relation $y_{2}=7 y_{1}$ and a presentation $H_{1}\left(M_{2}\right)=\left\langle y_{1} \mid y_{1}^{16}\right\rangle=\mathbf{Z} / 16 \mathbf{Z}$.

Let $\zeta$ (resp. $\xi$ ) be a primitive 16-th root of unity and let $\varphi: \mathbf{Z}\left[H_{1}\left(M_{1}\right)\right] \rightarrow \mathbf{Q}(\zeta)$ (resp. $\left.\psi: \mathbf{Z}\left[H_{1}\left(M_{2}\right)\right] \rightarrow \mathbf{Q}(\xi)\right)$ be a ring homomorphism such that $\varphi\left(x_{1}\right)=\zeta\left(\right.$ resp. $\left.\psi\left(y_{1}\right)=\xi\right)$. Set $\zeta_{i}:=\varphi\left(x_{i}\right)$ (hence $\zeta_{1}=\zeta$ and $\zeta_{2}=\zeta^{7}$ ) and $\xi_{i}=\psi\left(y_{i}\right)$ (hence $\xi_{1}=\xi$ and $\xi_{2}=\xi^{7}$ ) for $i=1,2$.

We first compute the torsion $\tau^{\varphi}\left(M_{1},\left[\mathcal{V}_{1}\right]\right)$. Since $\varphi \circ \operatorname{proj}\left(\frac{\partial}{\partial x_{1}}\left(R_{1}\right)\right)=1+\zeta_{1} \zeta_{2}^{-1}+$ $\zeta_{1}^{2} \zeta_{2}^{-4}=1+\zeta^{6}+\zeta^{10}$, the boundary operators are

$$
\partial_{2}^{\varphi}=\left(\begin{array}{c}
\zeta^{7}-1 \\
\zeta-1
\end{array}\right), \quad \partial_{1}^{\varphi}=\left(\begin{array}{cc}
1+\zeta^{6}+\zeta^{10} & * \\
* & *
\end{array}\right), \quad \partial_{0}^{\varphi}=\left(\begin{array}{cc}
\zeta-1 & \zeta^{7}-1
\end{array}\right) .
$$


Set $c_{3}=\{\hat{b}\}, c_{2}=\left\{\hat{d}_{1}, \hat{d}_{2}\right\}, c_{1}=\left\{\hat{h}_{1}, \hat{h}_{2}\right\}, c_{0}=\{\hat{c}\}$ and $b_{3}=\{\hat{b}\}, b_{2}=\left\{\hat{d}_{1}\right\}, b_{1}=\left\{\hat{h}_{2}\right\}$, $b_{0}=\emptyset$. Then

$$
\tau^{\varphi}\left(M_{1},\left[\mathcal{V}_{1}\right]\right)=\frac{\left[\partial^{\varphi}\left(b_{2}\right) b_{1} / c_{1}\right]}{\left[\partial^{\varphi}\left(b_{1}\right) b_{0} / c_{0}\right]\left[\partial^{\varphi}\left(b_{3}\right) b_{2} / c_{2}\right]}=\frac{1+\zeta^{6}+\zeta^{10}}{(\zeta-1)\left(\zeta^{7}-1\right)} \in \mathbf{Q}(\zeta)^{\times} / \pm 1 .
$$

Let us next compute the torsion $\tau^{\psi}\left(M_{2},\left[\mathcal{V}_{2}\right]\right)$, where $\mathcal{V}_{2}$ is the vector field on $M_{2}$ accompanying the DS-diagram. Then we have $\psi \circ \operatorname{proj}\left(\frac{\partial}{\partial y_{2}}\left(R_{1}^{\prime}\right)\right)=-\xi_{1} \xi_{2}^{-1}\left(1+\xi_{1} \xi_{2}^{-1}+\xi_{1}^{4} \xi_{2}^{-2}\right)=$ $-\xi^{10}\left(1+\xi^{6}+\xi^{10}\right)$. Hence the boundary operators are

$$
\partial_{2}^{\psi}=\left(\begin{array}{l}
\xi^{-1}-1 \\
\xi^{-7}-1
\end{array}\right), \partial_{1}^{\psi}=\left(\begin{array}{c}
* \\
* \\
-\xi^{10}\left(1+\xi^{6}+\xi^{10}\right) *
\end{array}\right), \partial_{0}^{\psi}=\left(\xi-1 \xi^{7}-1\right) .
$$
$b_{0}^{\prime}=\emptyset$.

Set $c_{3}^{\prime}=\{\hat{b}\}, c_{2}^{\prime}=\left\{\hat{d}_{1}, \hat{d}_{2}\right\}, c_{1}^{\prime}=\left\{\hat{h}_{1}, \hat{h}_{2}\right\}, c_{0}^{\prime}=\{\hat{c}\}$ and $b_{3}^{\prime}=\{\hat{b}\}, b_{2}^{\prime}=\left\{\hat{d}_{1}\right\}, b_{1}^{\prime}=\left\{\hat{h}_{1}\right\}$,

$$
\tau^{\psi}\left(M_{2},\left[\mathcal{V}_{2}\right]\right)=\frac{\left[\partial^{\psi} b_{2}^{\prime} b_{1}^{\prime} / c_{1}^{\prime}\right]}{\left[\partial^{\psi} b_{1}^{\prime} b_{0}^{\prime} / c_{0}^{\prime}\right]\left[\partial^{\psi} b_{3}^{\prime} b_{2}^{\prime} / c_{2}^{\prime}\right]}=\frac{\xi\left(1+\xi^{6}+\xi^{10}\right)}{(\xi-1)\left(\xi^{7}-1\right)} \in \mathbf{Q}(\xi)^{\times} / \pm 1 .
$$

Assume that $\left(M_{1},\left[\mathcal{V}_{1}\right]\right) \cong\left(M_{2},\left[\mathcal{V}_{2}\right]\right)$, that is, there exists a diffeomorphism $f: M_{2} \rightarrow$ $M_{1}$ such that $f_{*}\left(\mathcal{V}_{2}\right)$ and $\mathcal{V}_{1}$ are homologous in the class of non-singular vector fields on $M_{1}$. Then there is an integer $m(1 \leq m<16)$ coprime to 16 such that $\varphi \circ f_{\#}\left(y_{1}\right)=\zeta^{m}$ and that

$$
\frac{1+\zeta^{6}+\zeta^{10}}{(\zeta-1)\left(\zeta^{7}-1\right)}=\frac{\zeta^{m}\left(1+\zeta^{6 m}+\zeta^{10 m}\right)}{\left(\zeta^{m}-1\right)\left(\zeta^{7 m}-1\right)} \in \mathbf{Q}(\zeta)^{\times} / \pm 1
$$

We can assume without loss of generality that $\zeta=\exp \left(\frac{2 \pi \sqrt{-1}}{16}\right)$. We check which $m \in$ $\{1,3,5,7,9,11,13,15\}$ satisfies the formula (1).

In the case where $m=1, m=7, m=9$ and $m=15$, the right side of (1) are $\frac{\zeta\left(1+\zeta^{6}+\zeta^{10}\right)}{(\zeta-1)\left(\zeta^{7}-1\right)}, \frac{\zeta^{7}\left(1+\zeta^{6}+\zeta^{10}\right)}{(\zeta-1)\left(\zeta^{7}-1\right)}, \frac{\zeta\left(1+\zeta^{6}+\zeta^{10}\right)}{(\zeta-1)\left(\zeta^{7}-1\right)}$ and $\frac{\zeta^{7}\left(1+\zeta^{6}+\zeta^{10}\right)}{(\zeta-1)\left(\zeta^{7}-1\right)}$, respectively. Since the complex number $\frac{1+\zeta^{6}+\zeta^{10}}{(\zeta-1)\left(\zeta^{7}-1\right)}$ is not zero, and since $\arg (\zeta)$, and $\arg \left(\zeta^{7}\right)$ are not equal to 0 modulo $\pi$, we get $\arg \left(\frac{1+\zeta^{6}+\zeta^{10}}{(\zeta-1)\left(\zeta^{7}-1\right)}\right) \neq \arg \left(\frac{\zeta^{11 m}\left(1+\zeta^{6 m}+\zeta^{10 m}\right)}{\left(\zeta^{m}-1\right)\left(\zeta^{7 m}-1\right)}\right)$ modulo $\pi$. Therefore in this case, (1) is not formulated. In the other cases, the right side of $(1)$ is $\frac{\zeta^{m}\left(1+\zeta^{2}+\zeta^{14}\right)}{\left(\zeta^{3}-1\right)\left(\zeta^{5}-1\right)}$.

$$
\begin{gathered}
\left|\frac{1+\zeta^{6}+\zeta^{10}}{(\zeta-1)\left(\zeta^{7}-1\right)}\right|=\frac{\left|1+\zeta^{6}+\zeta^{10}\right|}{|\zeta-1|\left|\zeta^{7}-1\right|}=\frac{\sqrt{2}-1}{4 \sin \frac{\pi}{16} \sqrt{1-\sin ^{2} \frac{\pi}{16}}}=0.541196 \cdots \\
\left|\frac{1+\zeta^{2}+\zeta^{14}}{\left(\zeta^{3}-1\right)\left(\zeta^{5}-1\right)}\right|=\frac{\left|1+\zeta^{2}+\zeta^{14}\right|}{\left|\zeta^{3}-1\right|\left|\zeta^{5}-1\right|}=\frac{\sqrt{2}+1}{4 \sin \frac{3 \pi}{16} \sqrt{1-\sin ^{2} \frac{3 \pi}{16}}}=0.785694 \cdots
\end{gathered}
$$



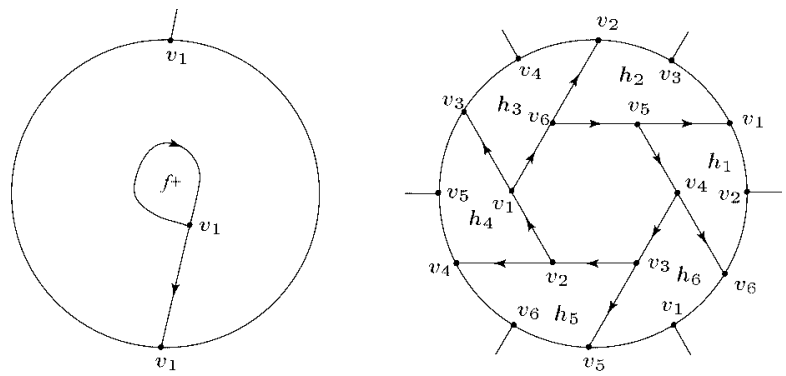

FIGURE 16. The Hopf flow on $S^{3}$ and the 6-fold cyclic branched covering space $M$.

Hence we get in this case $\left|\frac{1+\zeta^{6}+\zeta^{10}}{(\zeta-1)\left(\zeta^{7}-1\right)}\right| \neq\left|\frac{\zeta^{11 m}\left(1+\zeta^{6 m}+\zeta^{10 m}\right)}{\left(\zeta^{m}-1\right)\left(\zeta^{7 m}-1\right)}\right|$. This means there is no integer which satisfies the formula (1). This is a contradiction. Therefore we can conclude $\left(M,\left[\mathcal{V}_{1}\right]\right) \nsucceq\left(M,\left[\mathcal{V}_{2}\right]\right)$ although $M_{1}$ is diffeomorphic to $M_{2}$.

8.3. Cyclic branched covering Let $\mathcal{V}_{0}$ be the Hopf vector field in the 3 -sphere $S^{3}$. The diagram of $\left(S^{3}, \mathcal{V}_{0}\right)$ is shown in the left-hand side of Figure 16, and the knot $\mathrm{K}$ is the dual loop $f^{*}$ of the face $f$. Consider the 6-fold cyclic covering space $M$ branched over $K \subset S^{3}$ and the non-singular vector field $\mathcal{V}$ on $M$ induced from $\mathcal{V}_{0}$. Note that the flow $\mathcal{V}$ defines the Seifert fibration of $M$. The homology group $H_{1}(M)$ can be presented as follows:

$$
\left\langle x_{i}(1 \leq i \leq 6) \mid x_{i}-x_{i+1}-x_{i+5}(1 \leq i \leq 6)\right\rangle=\mathbf{Z} x_{1} \oplus \mathbf{Z} x_{2}=\mathbf{Z} \oplus \mathbf{Z},
$$

here the suffixes are mod 6. Let $\varphi: \mathbf{Z}\left[H_{1}(M)\right]=\mathbf{Z}[\mathbf{Z} \oplus \mathbf{Z}] \rightarrow Q(\mathbf{Z} \oplus \mathbf{Z})$ be the canonical map for the maximal abelian torsion and set $t_{1}=\varphi\left(x_{1}\right), t_{2}=\varphi\left(x_{2}\right)$, see [17]. By the same argument in the above examples, we get the presentation matrix of the boundary operators:

$$
\begin{aligned}
& \partial_{2}^{\varphi}=\left(\begin{array}{c}
t_{1}^{-1}-1 \\
t_{2}^{-1}-1 \\
t_{1} t_{2}^{-1}-1 \\
t_{1}-1 \\
t_{2}-1 \\
t_{1}^{-1} t_{2}-1
\end{array}\right), \quad \partial_{1}^{\varphi}=\left(\begin{array}{cccccc}
1 & -t_{1}^{-1} t_{2} & 0 & 0 & 0 & -1 \\
-1 & 1 & t_{1}^{-1} & 0 & 0 & 0 \\
0 & -1 & 1 & t_{2}^{-1} & 0 & 0 \\
0 & 0 & -1 & 1 & -t_{1} t_{2}^{-1} & 0 \\
0 & 0 & 0 & -1 & 1 & -t_{1} \\
-t_{2} & 0 & 0 & 0 & -1 & 1
\end{array}\right),
\end{aligned}
$$

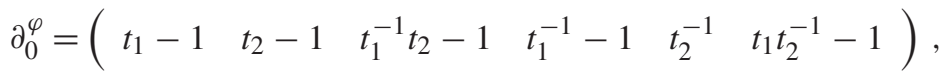


and the Reidemeister-Turaev torsion is

$$
\tau^{\varphi}(M,[\mathcal{V}])= \pm \frac{\operatorname{det}\left(\begin{array}{ccccc}
1 & t_{1}^{-1} & 0 & 0 & 0 \\
-1 & 1 & t_{2}^{-1} & 0 & 0 \\
0 & -1 & 1 & -t_{1} t_{2}^{-1} & 0 \\
0 & 0 & -1 & 1 & -t_{1} \\
0 & 0 & 0 & -1 & 1
\end{array}\right)}{\left(t_{1}^{-1}-1\right)\left(t_{1}-1\right)}= \pm \frac{\left(1-t_{1}^{-1}\right)\left(1-t_{1}\right)}{\left(t_{1}^{-1}-1\right)\left(t_{1}-1\right)}= \pm 1
$$

This implies the Seiberg-Witten invariant $S W_{M}([\mathcal{V}])$ of the $\operatorname{Spin}^{c}$ structure $[\mathcal{V}]$ is \pm 1 , following the relation $S W_{M}= \pm T_{M}$, where $T_{M}: \operatorname{Eul}(M) \rightarrow \mathbf{Z}$ is the torsion function, by Turaev $[15,16]$.

8.4. Torsions of standard $\operatorname{Spin}^{c}$ structures. The article [13] introduced an algorithm to obtain a DS-diagram of a standard vector field on an arbitrary closed Seifert fibered 3manifold starting from its Seifert invariant. The algorithm is based on the fact that any Seifert fibered manifold is constructed by gluing pieces each of which is homeomorphic to either $\left(S^{2} \backslash \bigsqcup_{i=1}^{3} \operatorname{Int} D_{i}\right) \times S^{1},\left(\left(S^{1} \times S^{1}\right) \backslash \bigsqcup_{i=1}^{3} \operatorname{Int} D_{i}\right) \times S^{1}$ or a fibered torus, where $D_{1}, D_{2}$ and $D_{3}$ are mutually disjoint closed disk in the surface. Then a DS-diagram is obtained by gluing the diagrams corresponding to the pieces. Combining this result and our above construction, we have the following:

COROLlARY 8.2. We can compute the Reidemeister-Turaev torsion of a closed oriented Seifert fibered manifold $M\left(F, b ;\left(p_{1}, q_{1}\right), \ldots,\left(p_{n}, q_{n}\right)\right)$ with a standard Spin ${ }^{-}$ structure in an algorithmic way.

We give an example. Consider the Seifert fibered manifold $M:=M\left(S^{2},-1 ;(3,1)\right.$, $(5,1),(7,1))$, i.e. the Seifert fibered manifold with base manifold $S^{2}$, obstruction class -1 and singular fibers of types $(3,1),(5,1)$ and $(7,1)$. Let $\mathcal{V}_{s t}$ be a standard flow on $M$. Then the DS-diagram of $\left(M,\left[\mathcal{V}_{\mathrm{st}}\right]\right)$ shown in 17 is obtained by using the algorithm of [13], see also [3, Theorem 4.3]. The fundamental group of the manifold $M$ has the presentation $\left\langle x_{1}, x_{2}, x_{3} \mid x_{1} x_{3} x_{2}^{-2}, x_{2} x_{1} x_{3}^{-4}, x_{3} x_{2} x_{1}^{-6}\right\rangle$ and hence we get $H_{1}(M)=\left\langle x_{1}, x_{2}, x_{3}\right| x_{1}+x_{2}-$ $\left.4 x_{3},-6 x_{1}+x_{2}+x_{3}, x_{1}-2 x_{2}+x_{3}\right\rangle=\left\langle x_{1} \mid x_{1}^{34}\right\rangle=\mathbf{Z} / 34 \mathbf{Z}, x_{2}=25 x_{1}$ and $x_{3}=15 x_{1}$.

Let $\zeta$ be a primitive 34-th root of unity and let $\varphi: \mathbf{Z}\left[H_{1}(M)\right] \rightarrow \mathbf{Q}(\zeta)$ be a ring homomorphism such that $\varphi\left(x_{1}\right)=\zeta$. The boundary operators of the twisted chain complex $C^{\varphi}(M)$ are given by:

$$
\partial_{2}^{\varphi}=\left(\begin{array}{c}
\zeta^{25}-1 \\
\zeta^{15}-1 \\
\zeta-1
\end{array}\right), \partial_{1}^{\varphi}=\left(\begin{array}{ccc}
1 & \zeta^{25} & * \\
-\left(1+\zeta^{25}\right) & 1 & * \\
* & * & *
\end{array}\right), \quad \partial_{0}^{\varphi}=\left(\zeta-1 \zeta^{25}-1 \zeta^{15}-1\right)
$$




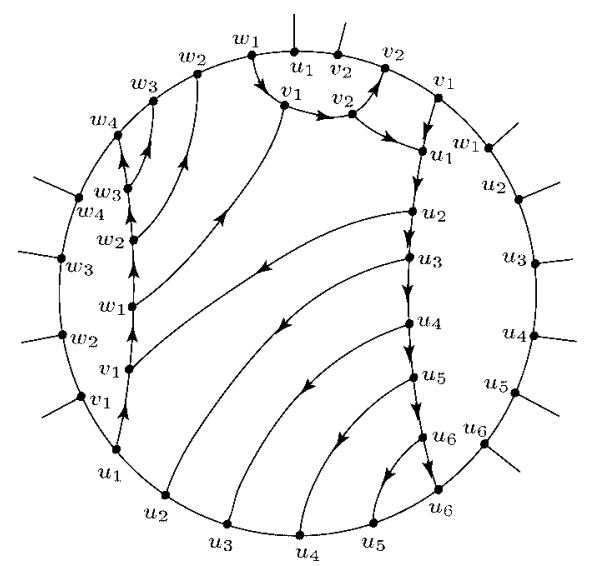

FIGURE 17. DS-diagram of $\left(M\left(S^{2},-1 ;(3,1),(5,1),(7,1)\right),\left[\mathcal{V}_{s t}\right]\right)$.

Due to Theorem 7.8, we get the Reidemeister-Turaev torsion

$$
\tau^{\varphi}\left(M,\left[\mathcal{V}_{s t}\right]\right)= \pm \frac{\operatorname{det}\left(\begin{array}{cc}
1 & \zeta^{25} \\
-\left(1+\zeta^{25}\right) & 1
\end{array}\right)}{(\zeta-1)\left(\zeta^{15}-1\right)}= \pm \frac{1+\zeta^{16}+\zeta^{25}}{(\zeta-1)\left(\zeta^{15}-1\right)} \in \mathbf{Q}(\zeta)^{\times} / \pm 1
$$

ACKNOWLEDGEMENT. I would like to thank Ippei Ishii for invaluable advice. I am also greatful to Hiroshi Goda, Masaharu Ishikawa, Teruaki Kitano, Takayuki Morifuji for helpful conversations. The author is supported by Research Fellowships of the Japan Society for the Promotion of Science for Young Scientists.

\section{References}

[1] R. Benedetti and C. Petronio, Branched Standard Spines of 3-manifolds, Springer "Lecture Notes in Mathematics 1653", 1997.

[2] R. Benedetti and C. Petronio, Reidemeister-Turaev torsion of 3-dimensional Euler structures with simple boundary tangency and pseudo-Legendrian knots, Manuscripta Math. 106 (2001), 13-74.

[ 3 ] M. ENDOH and I. IshiI, A new complexity for 3-manifolds, Japanese J. Math. 31 (2005), 131-156.

[ 4 ] H. IKEDA, DS-diagrams with E-cycle, Kobe J. Math. 3 (1986), 103-112.

[ 5 ] I. IsHII, Flows and spines, Tokyo J. Math. 9 (1986), 505-525.

[ 6] I. ISHII, Combinatorial construction of a non-singular flow on a 3-manifold, Kobe J. Math. 3 (1987), $201-208$.

[ 7 ] Y. KoDA, A Heegaard-type presentation of branched spines and Reidemeister-Turaev torsion, preprint.

[ 8 ] G. Meng and C. H. Taubes, $\underline{S W}=$ Milnor torsion, Math. Res. Lett. 3 (1996), 137-147.

[9] L. I. NiCOLAESCU, The Reidemeister Torsion of 3-Manifolds, Walter de Gruyter, 2003.

[10] P. OzSVÁth and Z. SZABó, Holomorphic disks and topological invariants for closed 3-manifolds, Ann. of Math. 159 (2004), no. 3, 1027-1158.

[11] P. OZSVÁtH and Z. SZABÓ, Holomorphic disks and 3-manifold invariants: properties and applications, Ann. of Math. 159 (2004), no. 3, 1159-1245.

[12] D. Rolfsen, Knots and Links, Math. Lect. Ser. 7, Publish or Perish, Berkeley, Carifornia, 1976. 
[13] T. TAniguchi, K. Tsuboi and M. Yamashita, Systematic singular triangulations for all Seifert manifolds, Tokyo J. Math. 28 (2005), 539-581.

[14] V. TURAEV, Euler structure, nonsingular vector fields, and Reidemeister-type torsions, Math. USSR-Izv. 34 (1990), 627-662.

[15] V. TuRAeV, Torsion invariants of $\operatorname{Spin}^{c}$-structures on 3-manifolds, Math. Res. Lett. 4 (1997), 679-695.

[16] V. TuraEv, A combinatorial formulation for the Seiberg-Witten invariants of 3-manifolds, Math. Res. Lett. 5 (1998), 583-598.

[17] V. TURAEV, Introduction to Combinatorial Torsion, Birkhäuser, 2001.

[18] V. TuRAeV, Torsions of 3-dimensional Manifolds, Birkhäuser, 2002.

Present Address:

DEPARTMENT OF MATHEMATICS,

KEIO UNIVERSITY,

HIYOSHI, KOHOKU-KU, YOKOHAMA, 223-8522 JAPAN.

e-mail:koda@math.keio.ac.jp 